\title{
Obesity-induced astrocyte dysfunction impairs heterosynaptic plasticity in the
} orbitofrontal cortex

Benjamin K. Lau ${ }^{1}$, Ciaran Murphy-Royal ${ }^{1}$, Manpreet Kaur ${ }^{1}$, Min Qiao ${ }^{1}$, Grant R. Gordon ${ }^{1}$, Jaideep S. Bains ${ }^{1}$, Stephanie L. Borgland ${ }^{1,2}$

${ }^{1}$ Department of Physiology and Pharmacology

Hotchkiss Brain Institute

The University of Calgary

3330 Hospital Dr. NW

Calgary, Alberta T2N 4N1 Canada

${ }^{2}$ Corresponding author

s.borgland@ucalgary.ca 


\section{SUMMARY}

Overconsumption of palatable, energy dense food is considered a key driver of the obesity pandemic. The orbitofrontal cortex (OFC) is critical for reward valuation of gustatory signals, yet how the OFC adapts to obesogenic diets is poorly understood. Here we show that an impairment of astrocyte glutamate clearance following extended access to a cafeteria diet reduces GABA release onto layer II/III pyramidal neurons in the lateral OFC in rats. This decrease in GABA tone is due to an increase in extrasynaptic glutamate, which activates metabotropic glutamate receptors (mGluR5) and liberates endocannabinoids. In obese rats, these synaptic impairments were rescued by the nutritional supplement, $\mathrm{N}$-acetylcysteine, which restores glutamate homeostasis. Together, our findings indicate that obesity targets astrocytes to disrupt the delicate balance between excitatory and inhibitory transmission in the lateral OFC.

\section{HIGHLIGHTS:}

- Synaptic plasticity within the OFC is disrupted with diet-induced obesity.

- Diet-induced obesity induces hypertrophic astrocytes in the OFC.

- Failure of astrocytes to clear synaptic glutamate drives endocannabinoid-mediated inhibitory long-term depression of OFC neurons.

- Astrocytic glutamate transporter function is restored with NAC, which rescues synaptic plasticity.

KEY WORDS: Orbitofrontal cortex, diet-induced obesity, synaptic transmission, GABA, glutamate astrocytes, endocannabinoid, $\mathrm{N}$-acetylcysteine 


\section{INTRODUCTION}

Obesity is characterized by a disruption in energy balance, in which intake of energy far exceeds its output (Spiegelman and Flier, 2001). One of the key contributors to excess energy intake is overeating, which is especially pertinent in modern times given the abundance of palatable, hypercaloric food. Furthermore, once obesity is established it is highly persistent, such that weight loss is rarely maintained (Hall and Guo, 2017). A hypothesis underlying the lasting effects of obesity is that adaptive plasticity occurs in neural circuits that cause animals to defend elevated body weight. Neural plasticity within hypothalamic and mesolimbic systems has been well characterized in regards to the mechanisms associated with feeding regulation, energy homeostasis as well as persistent adaptations following obesity (Matikainen-Ankney and Kravitz, 2018; Thoeni et al., 2020). However, there is growing evidence implicating cortical systems involved in decision-making (Lowe et al., 2019; Seabrook and Borgland, 2020). In the modern food environment, individuals must override automated responses to rewardingpredicting food cues and hyperpalatability in order to limit food intake. The orbitofrontal cortex (OFC) plays a key role in processing food-related signals (Jennings et al., 2019; Rolls, 2004; Seabrook and Borgland, 2020). The OFC integrates afferent and efferent projections from sensory, limbic and prelimbic regions (Ongür and Price, 2000) to guide decision-making associated with food intake. The lateral subdivision of the OFC (IOFC) sends strong projections to the dorsal medial and dorsal lateral striatum, and through these projections, the IOFC influences goal-directed behaviour (Gremel and Costa, 2013; Gremel et al., 2016; Ongür and Price, 2000). Disruption of these projections can induce habit-like behaviour, such that mice will continue to respond for food regardless of satiety (Gremel and Costa, 2013; Gremel et al., 2016). As such, the IOFC may be highly susceptible to perturbations associated with the obese state. Indeed, long-term exposure to an obesogenic diet induces structural and neuronal plasticity within the IOFC (Thompson et al., 2017). However, the upstream cellular mechanisms 
that underlie this effect have yet to be elucidated. Furthermore, there is little known about how overnutrition influences the metabolic homeostasis of neurons that governs appropriate synaptic function.

The endogenous cannabinoid (endocannabinoid) system plays an important role in regulating appetite and food intake (Di Marzo and Matias, 2005). Both exogenous and endogenous cannabinoids induce hyperphagia by activating cannabinoid type 1 (CB1) receptors within hypothalamic, mesolimbic and gustatory brain regions to respectively enhance hunger, desire and taste for food (Kirkham et al., 2002; Lau et al., 2017; Di Marzo, 2011; Soria-Gómez et al., 2014). Furthermore, obesity is typically associated with elevated levels of the major endocannabinoids, 2-arachidonoylglycerol (2-AG) and arachidonoyl ethanolamide (AEA) in both the brain and periphery (Gatta-Cherifi and Cota, 2016). Conversely, mice devoid of CB1 receptors are resistant to diet-induced obesity (Ravinet Trillou et al., 2004), highlighting the potential of pharmacologically blocking CB1 receptors to reduce weight gain. While an overactive endocannabinoid system is linked to diet-induced obesity, the precise mechanisms by which obesity alters endocannabinoid function in the cortex are not well understood. Given the importance of the endocannabinoid system in feeding and its direct modulation by diet, we hypothesized that overnutrition with palatable, energy dense food readily available for human consumption would influence endocannabinoid-mediated synaptic plasticity within the IOFC. 


\section{RESULTS}

\section{Extended access to a cafeteria diet alters endocannabionoid-mediated GABAergic} synaptic transmission.

To determine whether diet-induced obesity alters synaptic function in the IOFC, we gave 24h (extended), 1h (restricted), or no access (chow) to a cafeteria diet for 40 consecutive days (Figure 1A). Extended access to cafeteria diets are well known to result in diet-induced obesity in rats and we have previously characterized that weight gain is due to higher energy intake, predominantly derived from fat. Extended access to the cafeteria diet induces elevated plasma leptin and insulin levels consistent with obesity (Thompson et al., 2017). Consistent with previous reports (Johnson and Kenny, 2010; Rolls et al., 1980; Thompson et al., 2017), rats given extended access to a cafeteria diet gained significantly more weight than those with restricted or chow access (Figure 1B).

To compare basal GABAergic input to IOFC neurons between the dietary conditions, miniature inhibitory postsynaptic currents (mIPSCs) were recorded in layer II/III pyramidal neurons from extended, restricted and chow access rats. We found that the frequency, but not amplitude, of mIPSCs onto IOFC pyramidal neurons from extended access rats was significantly decreased compared to chow or restricted access rats (Figure 1C,D), consistent with a presynaptic mechanism of action reported previously (Thompson et al., 2017). Given that obesity is associated with an overactive endocannabinoid system (Gatta-Cherifi and Cota, 2016; Lau et al., 2017), we hypothesized that elevated levels of endocannabinoids may mediate the suppression of inhibition observed in the IOFC of extended access rats. If endocannabinoids are tonically present under basal conditions to suppress GABAergic transmission, then blockade of CB1 receptors should unmask a facilitation of inhibitory transmission. To test this idea, we examined the effect of the neutral CB1 receptor antagonist, NESS-0327 (0.5 $\mu \mathrm{M})$ on mIPSCs. We chose a neutral CB1 receptor antagonist as this would preclude effects due to constitutive 
receptor activity. Indeed, NESS-0327 $(0.5 \mu \mathrm{M})$ facilitated the rate of mIPSCs in IOFC pyramidal neurons from extended access rats to $126 \pm 5 \%$, but did not change that of restricted (96 $\pm 5 \%$ ), or chow (101 $\pm 6 \%$ ) access rats (Figure 1E). NESS-0327 had no effect on the amplitude of mIPSCs in IOFC neurons from chow $(96 \pm 2 \%)$, restricted $(99 \pm 5 \%)$ or extended $(95 \pm 2 \%)$ access rats (Figure 1E). Together, this indicates the presence of a basal endocannabinoid tone suppressing GABA release onto IOFC neurons from extended, but not chow or restricted access rats. To confirm that this effect can also occur with action potential-driven activity, we repeated the experiment with electrically-evoked IPSCs. Similarly, NESS-0327 (0.5 $\mu \mathrm{M})$ application unmasked a facilitation of evoked IPSCs in IOFC neurons from extended access (133 $\pm 8 \%)$, but not chow $(94 \pm 6 \%)$ or restricted access rats $(104 \pm 5 \%$, Figure $1 \mathrm{~F}-\mathrm{H})$. The facilitation of evoked IPSC amplitude by NESS-0327 was abolished in slices pre-incubated with the diacyglycerol lipase inhibitor, tetrahydrolipstatin (THL, $10 \mu \mathrm{M}, 96 \pm 7 \%$, Figure $1 \mathrm{~F}-\mathrm{H}$ ), which prevents the biosynthesis of the endocannabinoid, 2-AG. This indicates that there is a 2-AGmediated endocannabinoid tone suppressing evoked inhibitory synaptic transmission onto IOFC pyramidal neurons in extended access rats.

\section{Endocannabinoid-mediated long-term depression is impaired in IOFC pyramidal neurons}

\section{of extended access rats.}

We next investigated whether the presence of an endocannabinoid tone onto IOFC pyramidal neurons of extended access rats was associated with impairments in synaptic plasticity. We first tested if inhibitory synapses onto IOFC pyramidal neurons undergo long-term depression (LTD) in diet-naïve rats (Figure S1A). We found that strong theta-burst stimulation (TBS) induced a long-lasting suppression of evoked IPSC amplitude onto IOFC pyramidal neurons (Figure S1B-D). This was associated with paired-pulse facilitation (Figure S1E), 
suggesting a presynaptic mechanism of action. Previous work in the visual (Jiang et al., 2010) and somatosensory (Zhao et al., 2015) cortex has demonstrated that TBS-LTD requires endocannabinoids. Therefore, we tested if the CB1 receptor antagonist, AM251 could block TBS-induced LTD in IOFC neurons. TBS in the presence of AM251 (3 $\mu \mathrm{M})$ inhibited LTD and induced a small facilitation of evoked IPSCs (Figure S1B-D). Given that many forms of endocannabinoid-mediated LTD depend on activation of extrasynaptic group 1 metabotropic glutamate receptors, we examined TBS-LTD in the presence of the mGluR5 antagonist, MTEP. MTEP abolished TBS-LTD of evoked IPSC amplitude of IOFC neurons (Figure S1B-D), indicating that this form of LTD requires activation of mGluR5 in addition to CB1 receptors. Confirming this, AM251 or MTEP inhibited the paired-pulse facilitation induced by TBS stimulation (Figure S1E).

Having established that inhibitory synapses in the IOFC can undergo endocannabinoidmediated LTD, we next examined if TBS-LTD was altered by diet-induced obesity (Figure 2A). Following TBS, a long-term suppression of evoked IPSC amplitude was observed in IOFC pyramidal neurons of chow and restricted access rats $(69 \pm 9 \%$ and $67 \pm 5 \%$ of baseline, respectively, Figure $2 \mathrm{~B}-\mathrm{C}$ ), and this was associated with an increase in paired-pulse ratio in chow access rats (Figure 2D). By contrast, TBS had no effect on evoked IPSCs in IOFC pyramidal neurons of extended access rats (98 $\pm 4 \%$ of baseline, Figure $2 B-D)$. Taken together, endocannabinoid-mediated LTD is impaired in the IOFC of obese rats and this effect was not due to exposure to the diet in the absence of obesity.

Given the link between Group 1 mGluRs and endocannabinoid signaling, we next investigated whether the obesity-induced endocannabinoid tone and impairment of TBS-LTD was mediated upstream by activation of mGluR1/5 (Figure 3A). Similar to TBS-LTD, the mGluR1/5 agonist, DHPG (50 $\mu \mathrm{M})$ produced an LTD of evoked IPSC amplitude of IOFC neurons 
from chow access rats, which was blocked in the presence of AM251 (Figure 3B-D). Consistent with a presynaptic effect, DHPG produced a paired-pulse facilitation, which was blocked by AM251 (Figure 3E). By contrast, in rats with extended access to a cafeteria diet, DHPG (50 $\mu \mathrm{M})$ had no effect on evoked IPSCs onto IOFC pyramidal neurons (Figure 3B-E). Together, these results indicate that mGluR1/5 activation induces an endocannabinoid-mediated LTD, and this plasticity is impaired in obese animals.

We next tested if the endocannabinoid tone previously revealed by a neutral CB1 receptor antagonist in obese animals could be blocked with an mGluR5 antagonist (Figure 3F). In the presence of MTEP, NESS-0327 $(0.5 \mu \mathrm{M})$ no longer produced a facilitation of evoked IPSC amplitude of IOFC neurons of extended access rats (Figure 3G), indicating the necessity of mGluR5 activation in mediating endocannabinoid tone. Furthermore, MTEP application alone mimicked the facilitation of evoked IPSCs observed with NESS-0327 (Figure 3H). Together, these results indicate that mGluR5 activation induces endocannabinoid tone, which may occlude the induction of endocannabinoid-mediated LTD in the IOFC of obese rats.

\section{An obesogenic diet produces a shift in glutamatergic regulation of excitatory synapses on IOFC pyramidal neurons.}

The obesity-induced alteration in mGluR5 signalling may be due to a change in receptor sensitivity, number, or an occlusion by enhanced levels of synaptic glutamate spilling over onto extrasynaptically located mGluR1/5s. To address this, we first examined basal glutamatergic synaptic input onto IOFC neurons of chow or extended access rats (Figure 4A). In the presence of $\operatorname{TTX}(0.5 \mu \mathrm{M})$, miniature excitatory postsynaptic current (mEPSC) amplitude was not changed between diet groups (Figure 4B,D). However, there was a significant reduction in the frequency of mEPSCs onto IOFC neurons of extended access rats compared to chow access rats (Figure 
4B,C), indicative of a decrease in presynaptic glutamate release. We additionally gauged synaptic glutamate levels by examining the effect of the low-affinity AMPA receptor antagonist, $\gamma$-DGG $(1 \mu \mathrm{M})$ on evoked AMPA EPSCs (Figure 4D). Y-DGG competes with ambient glutamate to bind synaptic AMPA receptors. Thus, the inhibition of evoked AMPA EPSC amplitude produced by $\gamma$-DGG will inversely correlate with synaptic levels of glutamate. Compared to IOFC neurons from chow access rats, $\gamma$-DGG produced significantly greater inhibition of evoked AMPA EPSC amplitude of IOFC neurons of extended access rats (Figure 4E,F), indicating decreased synaptic glutamate. To interrogate if this effect was due to an alteration in synaptic glutamate or a change in AMPA receptor number or function, we applied the high-affinity AMPA receptor antagonist, DNQX $(1 \mu \mathrm{M})$, which fully displaces ambient glutamate from binding to synaptic AMPA receptors. There was no difference in DNQX-induced inhibition of evoked AMPA EPSCs of IOFC neurons from chow or extended access rats (Figure 4G,H), which together with the pattern of $\gamma$-DGG inhibition suggest a lack of change in AMPA receptor number/function. Contrary to our prediction that synaptic glutamate spillover leads to mGluR5 activation, the above results suggest there is decreased synaptic glutamate release onto IOFC neurons from obese rats.

We next explored the possibility that mGluR5 receptors are activated by enhanced levels of extrasynaptic glutamate derived from a non-synaptic source. To indirectly interrogate levels of extrasynaptic glutamate, we induced spillover of glutamate onto extrasynaptic NMDA receptors under conditions of high activity (Figure 4I). Pharmacologically isolated NMDA EPSCs were evoked at $+40 \mathrm{mV}$ in response to a high frequency train of electrical stimulation $(5 \times 100 \mathrm{~Hz})$ (Figure 4I-K). To measure the duration of glutamate action at NMDA receptors, the half-decay time of evoked NMDA EPSCs was analyzed. We found that baseline decay time was significantly prolonged in IOFC neurons from extended access rats relative to chow access rats 
(Figure 4J,K), indicating elevated levels of glutamate in the extrasynaptic space. To indirectly assess changes in the efficacy of glutamate transport, we examined the action of the nonselective glutamate transport inhibitor, DL-TBOA (TBOA, $30 \mu \mathrm{M})$. In chow access rats, TBOA produced a $319 \pm 37 \%$ increase in decay time, but this facilitation was significantly reduced to $164 \pm 13 \%$ in IOFC neurons of extended access rats (Figure $4 \mathrm{~J}, \mathrm{~K}$ ). This reduced effect of TBOA in extended access rats suggests there is either a partial occlusion of NMDA receptors by the tonic presence of extrasynaptic glutamate and/or reduced efficacy of glutamate transporters.

Given the observed increase in extrasynaptic glutamate and decrease in synaptic glutamate, we next tested if this compartmentalization of glutamate was mechanistically linked. One possibility for decreased synaptic glutamate release is through autoinhibition via presynaptic Group II mGluR2/3 activation by extrasynaptic glutamate. To test this possibility, we examined the effect of the mGluR2/3 agonist, LY379268 on evoked AMPA EPSCs of IOFC neurons from chow or extended access rats (Figure 5A). LY379268 (100 nM) inhibited evoked EPSCs from chow access rats (Figure 5B-D), which was reversed following addition of the mGluR2/3 antagonist, LY341495 (1 $\mu \mathrm{M})$ (Figure 5B-D). Thus, glutamatergic synapses of OFC pyramidal neurons are sensitive to extrasynaptic inhibition via mGluR2/3 activation. Compared to chow access rats, LY379268 produced significantly less inhibition of EPSCs of IOFC neurons from extended access rats (Figure 5B-D), indicating possible occlusion of mGluR2/3 by an extrasynaptic glutamate tone. To test this, we examined the effect of LY341495 alone on evoked EPSCs of IOFC neurons from chow or extended access rats (Figure 5E). LY341495 (1 $\mu \mathrm{M})$ had no effect on evoked EPSCs of IOFC neurons from chow access rats, but significantly facilitated this response in extended access rats (Figure 5F-H). Together, these data suggest that in obese rats, there is a persistent suppression of synaptic glutamate release via tonic activation of presynaptic mGluR2/3 receptors by extrasynaptic glutamate. 


\section{An obesogenic diet induces hypertrophic astrocytes in the OFC.}

So far we have demonstrated that during obesity, IOFC pyramidal neurons receive enhanced glutamate from a non-synaptic source. Given that astrocytes account for the majority of glutamate transport in the brain, we surmised that an obesogenic diet impairs astrocytes and their ability to uptake glutamate. We first examined expression of the astrocyte marker, glial fibrillary acidic protein (GFAP) within the OFC of chow, restricted or extended access rats (Figure 6A). Compared to the OFC of chow or restricted access rats, we found an increase in the intensity and area of GFAP expression in the OFC from extended access rats (Figure 6AC,E,F). However, there was no change in the number of GFAP+ astrocytes (Figure 6D,G). To investigate this further, we examined the finer morphology of astrocytes using fluorescent twophoton microscopy (Figure 6H). OFC astrocytes were first labelled in-vitro with the astrocytespecific dye, SR-101 (20 $\mu \mathrm{M}, 20$ mins). Individual astrocytes were then patch-clamped under fluorescence and filled with the large fluorescent dye, FITC-dextran (3-5 kDa) (Figure 6H). Astrocytes from the IOFC of extended access rats $\left(4407 \pm 203 \mu \mathrm{m}^{2}\right)$ had significantly larger astrocyte territories compared to those from chow access rats $\left(3654 \pm 214 \mu \mathrm{m}^{2}\right)$ (Figure $\left.6 \mathrm{I}, \mathrm{J}\right)$. However, there was no change in branching indicated by the Schoenen ramification index (Figure 6K). Together, these data indicate that an obesogenic diet induces hypertrophy of OFC astrocytes.

We next explored whether hypertrophic astrocytes in the OFC were associated with impaired glutamate transporter $1(\mathrm{GLT}-1)$ function, a transporter responsible for approximately 90\% of glial glutamate reuptake in the cortex (Danbolt et al., 1992; Rothstein et al., 1994).

Patch-clamping from SR101-labelled IOFC astrocytes in ACSF containing caged RubiGlutamate $(30 \mu \mathrm{M})$, we evoked the focal action of glutamate via flash photolysis (473 nm LED, 2 
ms, Figure 7A). An astrocytic glutamate transporter current was isolated in the presence of synaptic blockers and by subtracting the residual current following application of TBOA ( $30 \mu \mathrm{M})$ (Figure 7B). Glutamate transporter currents of IOFC astrocytes from extended access rats were significantly smaller than those from chow access rats (Figure 7B,C). We further examined GLT1 protein expression with western blots, but observed no difference in GLT-1 expression between the IOFC of chow or extended access rats (Figure 7D,E). Together, these results indicate impairment in the function but not expression of GLT-1 in IOFC astrocytes from obese rats.

We next tested the hypothesis that impairment of astrocytic glutamate transport leads to an endocannabinoid-mediated long-term depression of GABAergic transmission (Figure 7F). Similar to TBS-induced LTD and mGluR-LTD, application of TBOA (30 $\mu \mathrm{M}, 10 \mathrm{~min}$ ) suppressed evoked IPSC amplitude in IOFC neurons from chow access rats, which persisted for at least 20 min after washout of the drug (Figure $7 \mathrm{G}, \mathrm{H})$. This long-lasting suppression of inhibition was blocked by pre-treatment with AM251 $(3 \mu \mathrm{M})$, indicating that this glutamate transporter-induced effect is endocannabinoid-mediated. Furthermore, in the presence of AM251, TBOA induced a significant facilitation of evoked IPSC amplitude (Figure $7 \mathrm{G}, \mathrm{H}$ ). The TBOA-induced suppression of inhibition was absent in IOFC neurons from extended access rats, consistent with an occlusion by extrasynaptic glutamate due to impaired transporter function (Figure 7G,H). Taken together, these results indicate that inhibition of astrocytic glutamate transport can influence IOFC inhibitory synapses via an endocannabinoid-mediated LTD, and this effect is impaired during diet-induced obesity.

\section{Restoration of glutamate homeostasis with $\mathrm{N}$-acetylcysteine restores the alterations in}

\section{GABAergic synaptic transmission.}


Because altered GABAergic synaptic transmission in IOFC pyramidal neurons from extended access rats is mediated upstream by impaired glutamate transport, we surmised that restoration of glutamate homeostasis in vitro could reverse the cascade of synaptic changes observed with diet-induced obesity. We tested the effect of $\mathrm{N}$-acetylcysteine (NAC), a cystine prodrug reported to enhance GLT-1 activity and restore extrasynaptic glutamate concentration (Kupchik et al., 2012; Reissner et al., 2015). Following a 90 min pre-treatment with NAC (500 $\mu \mathrm{M})$, glutamate transporter currents were significantly enhanced in astrocytes of extended access rats (Figure 8A,B), similar in magnitude to those observed in astrocytes of chow access rats (Figure $7 \mathrm{~B}, \mathrm{C})$. This was associated with a reduction in high frequency-evoked NMDA EPSC decay time and a restoration of the ability of TBOA to enhance this measure (Figure 8B). Taken together, NAC enhances the function of astrocytic glutamate transporters, leading to a reduction in extrasynaptic glutamate onto IOFC pyramidal neurons from obese rats.

We then tested whether NAC could abolish the mGluR5-induced endocannabinoid tone and restore LTD at inhibitory synapses. Pre-treatment with NAC prevented the facilitation of evoked IPSC amplitude induced by NESS-0327 (Figure 8C,D) and restored mGluR-LTD at inhibitory synapses onto IOFC neurons from extended access rats (Figure 8E, G). Consistent with this, NAC pretreatment also restored TBS-LTD at inhibitory synapses of IOFC pyramidal neurons from extended access rats (Figure 8F, G). To interrogate the involvement of extrasynaptic glutamate in the rescue of TBS-LTD, we applied the specific GLT-1 blocker, dihydrokainic acid (DHK). In the presence of NAC, DHK (200 $\mu \mathrm{M})$ blocked TBS-induced LTD (Figure 8F, G), indicating that NAC indeed restores extrasynaptic glutamate to rescue TBSinduced LTD. Finally, pre-treatment with NAC significantly enhanced the frequency of mIPSCs onto IOFC neurons from extended access rats, (Figure $8 \mathrm{H}$ ), similar to that observed in chow access rats (Figure 8H), without influencing mIPSC frequency onto IOFC neurons from chow 
access rats. Together, these data indicate that by restoring glutamate transporter function and extrasynaptic glutamate levels, NAC abolishes endocannabinoid tone and the occlusion of endocannabinoid-mediated LTD, thereby rescuing the impairment of GABAergic synaptic transmission in obese rats.

\section{DISCUSSION}

These observations provide a number of new insights that expand our understanding of how obesogenic diets alter endocannabinoid function in the brain. Of primary importance, these data identify a novel astrocyte-synaptic mechanism in the IOFC that becomes disrupted in obesity. Following long-term exposure to an obesogenic diet, IOFC astrocytes undergo hypertrophy and this is associated with an impairment of glutamate transport via GLT-1. This leads to enhanced extrasynaptic glutamate, which generates an endocannabinoid tone via mGluR5 activation to induce an LTD of inhibitory synaptic transmission onto IOFC pyramidal neurons. Importantly, this cascade of synaptic deficits could be reversed by restoring glutamate homeostasis with the nutritional supplement, NAC (Figure S2). The data presented here demonstrate that long-term exposure to an obesogenic diet induces heterosynaptic plasticity of excitatory and inhibitory synapses within the IOFC via a neuron-glia interaction.

\section{An obesogenic diet influences astrocyte function in the IOFC.}

Extended access to an obesogenic diet leads to a structural hypertrophy of astrocytes, whereby the length of their processes expanded by $\sim 20 \%$. This was accompanied by increased GFAP immunoreactivity, indicative of astrogliosis. Consumption of an obesogenic diet can induce astrogliosis in the hypothalamus as early as 3 days after exposure (Thaler et al., 2012) as well as after diet-induced obesity is established (Thaler et al., 2012; Zhang et al., 2017). 
Hypothalamic astrocytes can undergo shortening of astrocytic processes measured with GFAP immunostaining (Zhang et al., 2017). However, the large GFAP+ primary branches only account for $10-20 \%$ of the total volume of astrocyte arborisation (Bushong et al., 2002). Therefore, we verified our GFAP immunostaining with fluorescent dye-filling of astrocytes using two-photon microscopy. We observed lengthening of astrocytic processes without an increase of GFAP expressing cells in the IOFC. Given that the ability of astrocytes to impact synaptic function relies on their physical interaction with synapses (Papouin et al., 2017), changes in astrocyte territory are likely to modify basal synaptic activity. Indeed, we observed altered kinetics of extrasynaptic glutamate action in the IOFC of obese rats. This effect was not due to enhanced synaptic glutamate spillover, as we observed a decrease in presynaptic glutamate release. By contrast, enhanced extrasynaptic glutamate was likely due to a change in GLT-1 function. Consistent with this, astrocytic glutamate transporter currents were significantly reduced in the IOFC of obese rats. The non-selective glutamate transport blocker, DL-TBOA, abolished glutamate transporter currents in our astrocyte-patch experiments indicating that the astrocytic glutamate transporters, GLAST and/or GLT-1 were involved. Given the respective $\mathrm{EC}_{50}$ of DLTBOA for these transporters is $70 \mu \mathrm{M}$ and $6 \mu \mathrm{M}$ (Shimamoto et al., 2000), the $30 \mu \mathrm{M}$ concentration of DL-TBOA used in this study should have predominantly targeted GLT-1. Furthermore, GLAST is unlikely to play a role given its expression is mainly restricted to the cerebellum with little to no expression in the cortex (Rothstein et al., 1994). In addition to reduced GLT-1 function, we observed an increase in length of astrocytic processes with no change in GLT-1 protein expression, suggesting that expression of GLT-1 may be diluted along astrocytic processes in the IOFC of obese rats. In the obese condition, other studies have shown a reduction of GLT-1 expression (Linehan et al., 2018; Tsai et al., 2018) or an upregulation of GLT-1 in hypothalamic or hippocampal tissue (Cano et al., 2014; ValladolidAcebes et al., 2012). This discrepancy may be associated with the density of astrocytic GLT-1 
transporter expression in reference to altered astrocytic morphology (Zhang et al., 2017).

Astrocyte morphology can directly impact glutamate transport (Oliet et al., 2001) and GLT-1

diffuses rapidly within the membrane to influence EPSC kinetics (Murphy-Royal et al., 2015).

Thus, it is conceivable that diluted expression of GLT-1 on extended astrocytic processes in the OFC of obese rodents may have a negative impact on synaptic glutamate homeostasis by disconnecting glutamate reuptake from synaptic release.

\section{An obesogenic diet disrupts endocannabinoid-mediated inhibitory synaptic plasticity in} the IOFC.

Rats with extended access to a cafeteria diet exhibited decreased GABAergic synaptic transmission onto layer II/III pyramidal neurons within the IOFC, consistent with our previous findings (Thompson et al., 2017). This was likely due to a suppression in presynaptic GABA release, as we observed a change in the rate, but not amplitude of mIPSCs. Furthermore, this presynaptic inhibition was associated with the presence of endocannabinoid tone, as a CB1 receptor antagonist inhibited the obesity-induced reduction of mIPSC frequency and unmasked a facilitation of evoked IPSCs. The facilitation of inhibitory synaptic transmission was not due to constitutive activity of $\mathrm{CB} 1$ receptors, as we used a neutral $\mathrm{CB} 1$ receptor antagonist devoid of inverse agonist properties. This endocannabinoid-mediated suppression of inhibition was mediated upstream through activation of mGluR5 by extrasynaptic glutamate. Multiple lines of evidence support this notion. An mGluR5 antagonist blocked endocannabinoid-mediated TBSinduced LTD in naïve rats, and this effect was absent in obese rats. Moreover, application of an mGluR1/5 agonist induced an LTD in chow-fed, but not obese rats. Finally, in obese rats, application of the mGluR5 antagonist blocked endocannabinoid tone unmasked by the CB1 receptor antagonist, and mimicked this tone when applied alone. These data indicate that the 
endocannabinoid tone is produced upstream through activation of mGluR5, rather than an obesity-induced alteration of endocannabinoid synthetic or degradation enzymes.

Impairment in astrocytic glutamate reuptake influenced GABAergic synaptic transmission in the IOFC of obese rats. To confirm that astrocytic dysfunction is directly linked to an extrasynaptic glutamate driven endocannabinoid-mediated suppression of GABAergic transmission, we showed that blockade of GLT-1 with TBOA induced an LTD of inhibitory transmission in the IOFC of chow access rats. This was endocannabinoid-mediated as it was prevented in the presence of a CB1 receptor antagonist. Importantly, the TBOA-induced LTD was absent in IOFC neurons of obese rats, suggesting an occlusion by an existing extrasynaptic glutamate tone. Therefore, our results suggest that a failure in glutamate reuptake can indirectly modulate GABAergic transmission via recruitment of endocannabinoids. While synaptic glutamate spillover can drive endocannabinoid-mediated suppression of GABAergic synapses in neurons under physiological conditions (Chevaleyre and Castillo, 2004; Drew et al., 2008), to our knowledge, the present study is the first to demonstrate that astrocyte dysfunction can drive this effect during a pathophysiological state such as obesity.

There is considerable evidence linking diet to alterations in brain endocannabinoid function (Gómez-Pinilla, 2008). Importantly, endocannabinoids are synthesized from essential fatty acids which are strictly derived from dietary sources (Simopoulos, 2016; Spencer et al., 2017). A diet-deficient in omega-3 fatty acids impairs synaptic plasticity within a number of brain regions, and this is associated with behavioural impairments (Lafourcade et al., 2011; Manduca et al., 2017; Thomazeau et al., 2016). The cafeteria diet employed here was composed of approximately $50 \%$ fat from mostly saturated sources. While omega- 3 fatty acids were present in chow given to all diet groups, rats with restricted or extended access to the cafeteria consume significantly less chow (Thompson et al., 2017). Given that the synaptic impairments observed herein only occurred in rats with extended access to the diet, it is more likely that the obese 
state rather than the diet per se contributed to the synaptic changes within the IOFC.

There are multiple consequences of elevated endocannabinoid tone in the IOFC of obese mice. Dendritic spine density is reduced following chronic cannabinoid exposure (Chen et al., 2013; Rubino et al., 2009). Notably, we previously observed a reduction of spine density of IOFC pyramidal neurons in rats with extended access to a cafeteria diet (Thompson et al., 2017). Thus, the persistent endocannabinoid tone induced by the diet may influence morphological features of IOFC pyramidal neurons. Elevated endocannabinoid tone may also disrupt the interplay of synaptic excitation and inhibition influencing cortical processing. We demonstrated that TBS-induced LTD at inhibitory synapses in the IOFC requires endocannabinoids through a canonical mGluR1/5-mediated mechanism in chow fed or naïve rats. However, after diet-induced obesity, both mGluR1/5- and TBS-induced LTD were occluded. Suppressed inhibitory plasticity may disrupt the feedback and feed-forward circuits underlying the regulation of cortical network activity. Notably, deletion of CB1 receptors from OFC output neurons disrupts endocannabinoid regulation of terminals in the dorsal striatum, leading to habit formation (Gremel et al., 2016). Thus, disrupted endocannabinoid regulation of OFC principal output neurons may be important in driving inflexible behaviour.

\section{$\mathrm{N}$-acetylcysteine restores the diet-induced alterations in excitatory and inhibitory plasticity}

There is an increasing body of work indicating that NAC has efficacy in the treatment of neuropsychiatric conditions, including substance use disorder and compulsive behaviours (Baker et al., 2003; Brown et al., 2013; Grant et al., 2009, 2016; Moussawi et al., 2009). Furthermore, an accumulation of experimental evidence supports the therapeutic benefits of NAC treatment in obesity through its anti-inflammatory and antioxidant properties, as well as its ability to interfere with adipogenesis (Dludla et al., 2019). NAC can increase cystine-glutamate 
exchange and enhance glutathione synthesis, a potent antioxidant (McBean, 2002). In addition to activation of the cystine-glutamate exchanger, NAC appears to restore aberrant glutamate homeostasis associated with cocaine self-administration by enhancing glutamate transport via

GLT-1 (Kupchik et al., 2012; Reissner et al., 2015). Our data indicates that NAC can reverse the cellular alterations within the IOFC induced by long-term exposure to an obesogenic diet, suggesting possible therapeutic potential in reversing the synaptic deficits associated with obesity. While prior human and animal work has demonstrated efficacy of NAC in reducing weight gain (Dludla et al., 2019), whether it produces this effect via action within the OFC remains to be determined. Our findings have identified an extended cellular mechanism of action by NAC, which may additionally restore GABA homeostasis within the brain.

In conclusion, our findings provide a novel cellular mechanism by which an obesogenic diet alters inhibitory and excitatory transmission within the OFC. By altering astrocytes and their ability to transport glutamate, an obesogenic diet disrupts glutamate homeostasis, resulting in excess glutamate in the extrasynaptic space. This indirectly leads to an endocannabinoidmediated long-term suppression of GABAergic transmission. Together, this disinhibition may ultimately disrupt the excitatory-inhibitory balance in the OFC and resultant output from primary projection neurons of the OFC. This disruption in synaptic function may underlie behaviours associated with overeating, leading to a persistence of obesity. Importantly, these alterations in synaptic transmission are reversed by restoring glutamate homeostasis with NAC, highlighting the potential of this drug in reversing the synaptic dysregulation induced by obesity in the OFC.

\section{FUNDING AND DISCLOSURE}

This research was supported by a Canadian Institutes of Health Research operating grant (CIHR, FDN-147473) and a Canada Research Chair Tier 1 (950-232211) to SLB. Benjamin K 
Law was supported by postdoctoral awards from the Cumming School of Medicine (Eyes High) and Alberta Innovates Health Solutions. The authors declare no competing financial interests.

\section{ACKNOWLEDGEMENTS}

The authors would like to acknowledge the Hotchkiss Brain Institute optogenetic core facility and the advanced microscopy facility for their technical support.

\section{DATA ACCESSIBILITY}

Data will be made available upon request.

\section{AUTHOR CONTRIBUTIONS}

B.K.L. designed, performed and analyzed all electrophysiological experiments with supervision of S.L.B. C.M-R. performed astrocyte labeling experiments with supervision of G.G. and J.S.B. M.K. and M.Q. performed immunohistochemistry experiments and western blots under supervision of S.L.B. B.L.K. and S.L.B. wrote the manuscript. 


\section{FIGURE LEGENDS}

\section{Figure 1. Extended access to a cafeteria diet leads to an endocannabinoid-mediated} reduction in inhibitory GABAergic synaptic transmission onto IOFC pyramidal neurons.

(A) Schematic of experimental paradigm: Rats were given $24 \mathrm{~h}$ (extended), $1 \mathrm{~h}$ (restricted) or 0 h (chow) access to a cafeteria diet for 40 days. In-vitro patch-clamp electrophysiology was then conducted on layer II/III pyramidal neurons from brain slices containing the IOFC. (B) Change in body weight following 40 days access to either chow $(N=27)$, restricted ( $1 \mathrm{~h} /$ day; $N=27)$, or extended (24 h/day, $\mathrm{N}=38)$ access to a cafeteria diet. Rats with extended access to a cafeteria diet gain significantly more weight compared to those on chow $(P<0.001)$ or with restricted access $(P<0.001)$. One way ANOVA, $F(2,89)=68.49$, $P<0.001$, Tukey's posthoc tests. (C) Example traces of mIPSCs before (Baseline) and during application of the neutral CB1 receptor antagonist, NESS-0327 $(0.5 \mu \mathrm{M})$ of IOFC pyramidal neurons from chow, restricted or extended access rats. Scale bars: 50 pA, 100 ms.

(D) Basal rate (upper) and amplitude (lower) of mIPSCs onto IOFC neurons of chow ( $n=10 / 6)$, restricted $(n=14 / 7)$ or extended $(n=17 / 8)$ access rats. Rats with extended access to the cafeteria diet have decreased mIPSC rate compared to chow and restricted access rats (Oneway ANOVA, $F(2,38)=5.107, P=0.011$ with Tukey's multiple comparison test). There was no significant difference in mIPSC amplitude between diet groups $(F(2,38)=0.380, P=0.686)$.

(E) Percentage change in mIPSC rate and amplitude during application of NESS-0327 (0.5 $\mu \mathrm{M})$ in IOFC pyramidal neurons from chow $(n=8 / 5)$, restricted $(n=12 / 5)$ or extended $(n=13 / 8)$ access rats. NESS-0327 unmasks a facilitation of mIPSC rate in extended access rats $(\mathrm{P}<$

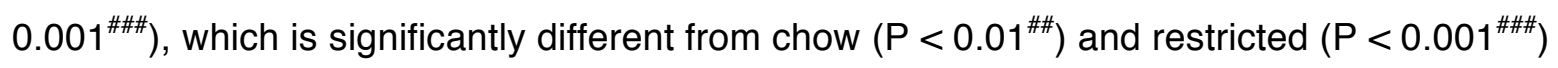
access rats $(F(2,30)=11.37, P=0.0002)$. NESS-0327 had no significant effect on amplitude $(F(2,30)=0.367, P=0.696)$. mIPSC rate of IOFC neurons from extended access rats was 
significantly greater than baseline $\left(t(12)=5.326, p=0.0002^{\star \star *}\right)$, whereas mIPSC rate from chow $(t(7)=0.212, p=0.838)$ and restricted access were not $(t(11)=0.682, p=0.509)$.

(F) Example traces of evoked IPSC amplitude before (black) and during application of NESS-

0327 in chow (top), restricted (middle), and extended (bottom) access rats. Scale bars: 100 pA, $10 \mathrm{~ms}$.

(G) Time course of evoked IPSC amplitude during application of NESS-0327 of IOFC pyramidal neurons from chow $(n=4 / 3)$, restricted $(n=5 / 4)$ and extended access $(n=7 / 4$ without THL, $n=$ 5/4 with $T H L)$ rats. Broken vertical lines indicate the time period analyzed in (G). There was a significant interaction of diet group $x$ timecourse $(F(87,527)=4.960, P<0.0001)$ and a main effect of diet $(F(3,19)=10.89, P=0.002$ (Mixed-effect model 2-way RM ANOVA).

(H) Bar graph quantifying the percentage change in evoked IPSC amplitude during application of NESS-0327 to IOFC pyramidal neurons from chow $(n=4 / 3)$, restricted $(n=5 / 4)$, or extended ( $n=7 / 4$ without THL, $n=5 / 4$ with THL) access rats. NESS-0327 unmasked a facilitation of evoked IPSC amplitude in IOFC pyramidal neurons of extended access rats $(P<0.05)$, which was blocked in the presence of an inhibitor of 2-AG synthesis, $T H L(P>0.05)(F(3,17)=7.445$, $P=0.0021)$. Evoked IPSC amplitude IOFC neurons from extended access rats was significantly greater than pre-NESS-0327 baseline $\left(t(6)=4.246, p=0.0054^{* *}\right.$, whereas evoked IPSCs from IOFC neurons of chow $(\mathrm{t}(3)=0.9659, \mathrm{P}=0.4054)$, restricted access $(\mathrm{t}(4)=0.9428, \mathrm{P}=0.3991)$ or extended access $+\mathrm{THL}(\mathrm{t}(4)=0.6231, \mathrm{P}=0.6231)$ were not.

Bars represent mean \pm s.e. $m$ with individual values overlaid.

Figure 2. Endocannabinoid-mediated inhibitory LTD of IOFC pyramidal neurons is impaired with extended, but not chow or restricted access to a cafeteria diet.

(A) Experimental schematic illustrating theta-burst stimulation (TBS) induced via electrical stimulation and its effect on evoked IPSCs. Stimulation consisted of 8 trains of TBS (5 sec 
intertrain interval). Each TBS train consisted of 10 bursts (200 ms interburst interval). Each burst consisted of 5 pulses at $100 \mathrm{~Hz}$.

(B) Time plot of evoked IPSC amplitude before and after TBS in IOFC neurons of chow $(\mathrm{n}=$ $11 / 9)$, restricted $(n=13 / 9)$ or extended $(n=11 / 7)$ access rats. Dotted vertical lines indicate the 25-30 min time period following TBS analyzed in (C). There was a significant interaction of diet group $x$ timecourse $(F(116,2053)=2.819, P<0.0001)$ and a main effect of diet $(F(2,44)=$ 11.00, $P=0.0001^{\star \star *}$ (Mixed-effect model 2-way RM ANOVA). Inset: Example traces of evoked IPSC amplitude before and after theta-burst stimulation (TBS) in IOFC pyramidal neurons from chow, restricted or extended access rats. Scale bars: 200 pA, 20 ms.

(C) Bar graph quantifying the percentage change in evoked IPSC amplitude 25-30 min following TBS in IOFC neurons from chow, restricted or extended access rats. TBS-LTD is present in IOFC neurons of chow $\left(P<0.01^{\# \#}\right)$ and restricted $\left(P<0.01^{\# \#}\right)$ access rats, but is impaired in extended access rats $(F(2,32)=7.511, P=0.002)$. Evoked IPSCs after TBS is significantly decreased from baseline in IOFC neurons from chow $\left(n=11 / 9, t(10)=3.495, P=0.0058^{\star \star \star}\right)$ and restricted access rats $\left(n=13 / 9, t(12)=6.637, P<0.0001^{\star \star \star \star}\right)$, but not extended $(n=11 / 7, t(10)$ $=0.3991, P=0.6982)$ access rats.

(D) Bar graph of the paired-pulse ratio of evoked IPSC amplitude before (Baseline) and after TBS (Post-TBS) of IOFC neurons of chow, restricted and extended access rats. A RM 2-way ANOVA indicates a significant diet $x \operatorname{LTD}$ interaction $(F(2,43)=5.087, P=0.010)$ with a main effect of TBS $(F(1,43)=8.537, P=0.0055)$. A Sidak's multiple comparison test reveals a significant effect of TBS on paired-pulse facilitation in IOFC neurons from chow $(n=11 / 9, P<$ $0.01)$, but not restricted $(n=13 / 9)$ or extended access rats $(n=11 / 7)$.

Bars represent mean \pm s.e. $m$ with individual values overlaid. 
Figure 3. Obesogenic diet-induced changes in endocannabinoid-mediated plasticity are mediated upstream by Group 1 mGluRs.

(A) Experimental schematic illustrating the effect of the mGluR1/5 agonist, DHPG on evoked IPSCs of IOFC neurons.

(B) Example traces of evoked IPSC amplitude before and after application of DHPG (50 $\mu \mathrm{M})$ in slices from chow, chow + AM251 (3 $\mu \mathrm{M}$, CB1 receptor antagonist) treated and extended access rats. Scale bars: $250 \mathrm{pA}, 25 \mathrm{~ms}$.

(C) DHPG induces an LTD in chow $(n=10 / 5)$ access rats, which is blocked in slices pre-treated with AM251 (3 $\mu M, n=15 / 5)$. However, DHPG does not induce LTD in extended $(n=12 / 6)$ access rats. Dotted vertical lines indicate the time period analyzed in (G). A Mixed-effect model 2-way RM ANOVA indicates a significant diet group $x$ LTD interaction $(F(78,1140)=4.684, P$ $<0.0001)$ with a main effect of $\operatorname{diet}(F(2,34)=12.85, P<0.0001)$ and a main effect of LTD $(F(5.110,149.4)=11.59, P<0.0001)$

(D) Bar graph quantifying the percentage inhibition of evoked IPSC amplitude by DHPG (15-20 min post-application) in IOFC pyramidal neurons of chow $(\mathrm{n}=8 / 5)$, chow $+\operatorname{AM} 251(3 \mu \mathrm{M}, \mathrm{n}=$ $7 / 5)$, or extended access rats $(n=8 / 6)$. DHPG produces a long-term suppression of evoked IPSC amplitude, which is blocked in the presence of AM251 and absent in extended access rats $(F(2,20)=23.70, P<0.0001$, Tukey's multiple comparison test: chow vs. chow + AM251,

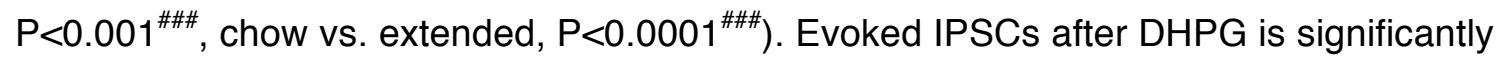
decreased from baseline in IOFC neurons from chow $\left(\mathrm{n}=8 / 5, \mathrm{t}(7)=7.37, \mathrm{P}=0.0002^{\star * \star}\right)$, but not in the presence of AM251 $(n=7 / 5, t(6)=0.923, P=0.392)$ nor in extended access rats $(n=8 / 6$, $t(7)=1.18, P=0.276)$. Some cells were excluded if they died before the analysis period.

(E) Bar graph of paired-pulse ratio of evoked IPSCs before and 15-20 min after application of DHPG on IOFC pyramidal neurons from chow $(n=9 / 5)$, chow + AM251 $(n=9 / 5)$ and extended access rats $(n=8 / 6)$. A 2-way RM ANOVA: Diet $x$ drug interaction: $(F(2,23)=2.690, P=0.089)$. 
A Sidak's multiple comparisons test indicates that DHPG elicits paired-pulse facilitation in IOFC neurons from chow $\left(\mathrm{P}<0.05^{\#}\right)$, but not in the presence of $\mathrm{AM} 251$, or in extended access rats. (F) Experimental schematic showing the effect of blocking CB1 receptors with NESS-0327 or mGluR5 with MTEP on evoked IPSCs of IOFC neurons from extended access animals.

(G) The facilitation of evoked IPSC amplitude by NESS-0327 $(0.5 \mu \mathrm{M})$ is blocked by the mGluR5 antagonist, MTEP in IOFC neurons from extended access rats $(n=7 / 5)$. Inset: Example traces of evoked IPSC amplitude before and during application of the neutral CB1 receptor antagonist, NESS-0327 from slices pre-incubated in the mGluR5 antagonist, MTEP from IOFC neurons of extended access rats. Scale bars: 250 pA, 25 ms.

(H) MTEP unmasks a facilitation of evoked IPSC amplitude, indicative of an mGluR5-mediated tone onto IOFC pyramidal neurons of extended access rats $(n=7 / 5)$. Bars represent mean \pm s.e.m with individual values overlaid. Inset: Example traces of evoked IPSC amplitude before and during application of MTEP. Scale bars: 250 pA, 25 ms.

\section{Figure 4: An obesogenic diet alters extrasynaptic and synaptic glutamate via impairment of astrocytic GLT-1.}

(A) Experimental schematic illustrating the recording of miniature excitatory postsynaptic currents (mEPSCs) in the presence of tetrodotoxin (TTX, $0.5 \mu \mathrm{M})$.

(B) Example traces of mEPSCs of IOFC pyramidal neurons from chow and extended access rats. Scale bars: $10 \mathrm{pA}, 0.1 \mathrm{~s}$.

(C) Frequency of mEPSCs of IOFC pyramidal neurons from extended ( $n=10 / 5)$ access rats is significantly reduced compared to that of chow $(n=7 / 4)$ access rats $\left(t_{(15)}=3.82, P=0.0017^{\# \#}\right)$. Inset,

(D) Amplitude of mEPSC is no different between IOFC pyramidal neurons of extended $(n=10 / 5)$ and chow $(n=7 / 4)$ access rats $\left(t_{(15)}=1.11, P=0.285\right)$. 
(E) Experimental schematic illustrating the effect of the low affinity, competitive AMPA receptor antagonist, $\gamma$-DGG on evoked EPSCs. $\gamma$-DGG competes with glutamate to bind with AMPA receptors. The degree of AMPA EPSC inhibition produced by $\gamma$-DGG is inversely correlated with synaptic glutamate levels.

(F) Example traces of evoked AMPA EPSCs before and during application of $\gamma$-DGG $(1 \mu M)$ in chow (scale bar: 500 pA, $25 \mathrm{~ms}$ ) and extended access rats (scale bar: 250 pA, $25 \mathrm{~ms}$ ).

(G) Time plot of evoked AMPA EPSC amplitude before and during $\gamma$-DGG application in IOFC pyramidal neurons of chow $(n=8 / 5)$ and extended $(n / N=9 / 7)$ access rats. A mixed-effects model $(R E M L)$ reveals a significant diet $x$ time interaction $(F(29,422)=3.135, P<0.0001)$, main effect of diet $\left(F(1,15)=7.683, P=0.0142^{\star}\right)$.

(H) Time plot evoked of evoked AMPA EPSC amplitude before and during application of the high-affinity AMPA receptor antagonist, DNQX $(10 \mu \mathrm{M})$ in IOFC neurons of chow $(n=8 / 5)$ or extended $(n=5 / 4)$ access rats. A mixed-effects model (REML) reveals a no diet $x$ time interaction $(F(29,290)=0.531, P=0.979)$, or main effect of diet $(F(1,11)=1.130, P=0.310)$. (I) Bar graph quantifying the percentage inhibition of AMPA EPSC amplitude in response to $\gamma$ DGG and DNQX in IOFC pyramidal neurons from chow or extended access rats. A 2-way ANOVA indicates a significant interaction of diet $x \operatorname{drug}(F(1,26)=4.45, P=0.447)$. A Sidak's multiple comparison test indicates that $\gamma$-DGG produces differential inhibition of evoked EPSC amplitude in IOFC neurons of chow compared to extended access rats $\left(P<0.05^{\#}\right)$.

(J) Experimental schematic illustrating the measurement of extrasynaptic glutamate via extrasynaptic NMDA receptors. Evoked NMDA EPSCs are isolated by recording at $+40 \mathrm{mV}$ in the presence of DNQX $(10 \mu \mathrm{M})$, picrotoxin $(100 \mu \mathrm{M})$ and strychnine $(1 \mu \mathrm{M})$. A high-frequency stimulation train $(5$ pulses $@ 100 \mathrm{~Hz}$ ) is used to elicit glutamate spillover into the extrasynaptic 
space. The effect of the glutamate transporter blocker, TBOA $(30 \mu \mathrm{M})$ on evoked NMDA EPSCs is examined.

(K) Example traces of evoked NMDA EPSCs in response to a $5 \times 100 \mathrm{~Hz}$ stimulus normalized to peak amplitude before and during application of the glutamate transport blocker, TBOA in IOFC neurons from chow or extended $(\mathrm{K})$ access rats. Artefacts have been minimized for clarity. Scale bars: $0.5,50 \mathrm{~ms}$.

(L) Half-decay time of evoked NMDA EPSCs during an initial baseline period (1 $\mathrm{min}$ ) immediately following whole-cell access to an IOFC pyramidal neuron. Half-decay time is significantly longer in IOFC pyramidal neurons of extended $(n / N=21 / 5)$ access rats compared to chow $(\mathrm{n} / \mathrm{N}=17 / 6)$ rats (Unpaired t-test, $\left.\mathrm{t}_{(36)}=2.89, \mathrm{P}=0.0065^{\# \#}\right)$.

(M) Change in decay time of evoked NMDA EPSCs in response to 10 min application of TBOA. Decay time is enhanced by TBOA in IOFC neurons of both chow $\left(n=9 / 4, t_{(8)}=5.956, P=\right.$ $\left.0.0003^{\star \star \star}\right)$ and extended access rats $\left(n=9 / 6, t_{(8)}=4.885, P=0.0012^{\star \star}\right)$ compared to baseline. However, this enhancement is significantly impaired in IOFC pyramidal neurons of extended access rats $\left(t_{(16)}=3.97, P=0.0011^{\# \#}\right)$.

Bars represent mean \pm s.e. $m$ with individual values overlaid.

Figure 5: Glutamatergic synapses of IOFC pyramidal neurons from extended access rats are tonically suppressed by glutamate acting via extrasynaptic mGluR2/3 receptors.

(A) Experimental schematic illustrating the effect of the mGluR2/3 agonist, LY379268 on evoked AMPA EPSCs.

(B) Example traces of evoked AMPA EPSCs before (baseline) and during application of LY379268 (100 nM) in IOFC neurons from chow or extended access rats. Scale bar: 500 pA, 10 ms. 
(C) Time course of evoked EPSC amplitude before and during application of LY379268 (100

$\mathrm{nM})$, and then after further addition of the mGluR2/3 receptor antagonist, LY341495 $(1 \mu \mathrm{M})$ on

IOFC neurons from chow $(n=11 / 4)$ or extended access rats $(n=10 / 4)$. Dotted vertical lines

show the time period analyzed in (C). There was a significant Diet x Time interaction, $F(29,494)$

$=4.097, \mathrm{P}<0.0001$ and $\mathrm{a}$ main effect of diet, $\mathrm{F}(1,18)=11.03, \mathrm{P}=0.0038^{\star \star}$.

(D) Bar graph quantifying the percentage inhibition of evoked EPSC amplitude by LY379268 in

Chow and Extended access rats. LY379268 produces significantly more inhibition of evoked

EPSC amplitude of IOFC neurons from chow compared to extended access rats. A mixed-

effects model (REML) indicated no diet $x$ drug interaction $(F(1,14)=0.887, P=0.362)$, but main

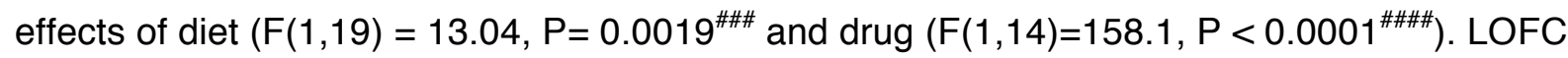

neurons from chow $\left(n=10 / 4, t_{(9)}=8.997, P<0.0001^{\star \star * \star}\right)$ or extended access rats $\left(n=11 / 4, t_{(10)}\right.$

$=12.06, P<0.0001^{\star \star \star \star}$ ) have LY379368-induced significantly decreased responses from

baseline.

(E) Experimental schematic illustrating the effect of the mGluR2/3 antagonist, LY341495 on

evoked AMPA EPSCs.

(F) Example traces of evoked AMPA EPSCs before (baseline) and during application of

LY341495 (1 $\mu \mathrm{M})$ onto IOFC neurons from chow and extended access rats. Scale bars: 250 pA, $10 \mathrm{~ms}$.

(G) Time course of evoked EPSC amplitude before and during application of the mGluR2/3

antagonist, LY341495 (1 $\mu M)$ in IOFC neurons from chow $(n=7 / 4)$ and extended access rats $(n$ $=7 / 3)$. A mixed-effects model $(R E M L)$ indicates a significant diet $x$ time interaction $(F(24,296)=$

12.40, $P<0.0001)$ and a significant diet effect: $\left(F(1,13)=24.35, P=0.0003^{\star * *}\right)$.

(H) Bar graph quantifying the percentage facilitation of evoked EPSC amplitude by LY341495 in IOFC neurons from chow $(n=7 / 4)$ and extended $(n=7 / 3)$ access rats. There was a significant 
difference of LY341495 on EPSC amplitude between IOFC neurons from chow and extended

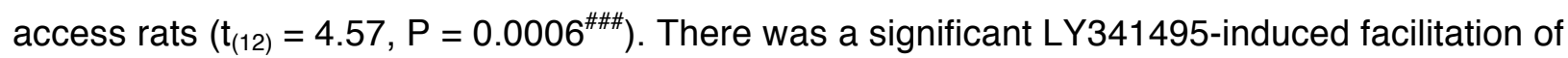
EPSCs over baseline of IOFC neurons from extended access rats $\left(t_{(6)}=5.424, P=0.0016^{\star \star}\right)$, but not chow access rats $\left(\mathrm{t}_{(6)}=0.6378, \mathrm{P}=0.5471\right)$.

Bars represent mean \pm s.e. $m$ with individual values overlaid.

Figure 6: Astrocytes are hypertrophic following exposure to an obesogenic diet

(A) Representative images illustrating GFAP expression in the IOFC of chow (upper), restricted (middle) and extended (lower) access rats. Scale bar, $50 \mu \mathrm{m}$.

(B) Quantification of percentage area of fluorescence of GFAP from slices containing the IOFC of chow ( $n=12$ slices $/ 5$ rats), restricted ( $n=16$ slices $/ 4$ rats) or extended ( $n=14 / 5$ rats). GFAP

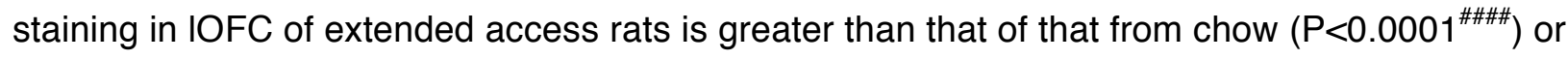
restricted access rats $\left(P<0.01^{\# \#}\right)$, One way ANOVA: $\left.F(2,44)=13.29, P<0.0001\right)$.

(C) Quantification of relative intensity units of GFAP fluorescence from slices containing the IOFC of chow ( $n=16$ slices $/ 5$ rats), restricted ( $n=17$ slices $/ 4$ rats) or extended $(n=18 / 5$ rats). GFAP staining in IOFC of extended access rats is greater than that of that from chow

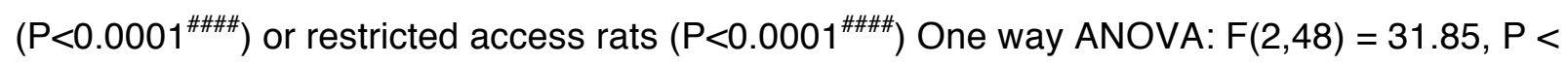
$0.0001)$.

(D) Quantification of the number of GFAP expressing cells from slices containing the IOFC of chow ( $n=12$ slices $/ 5$ rats), restricted ( $n=16$ slices $/ 4$ rats) or extended ( $n=14 / 5$ rats). There is no significant difference in the number of GFAP+ cells in IOFC of chow, restricted or extended access rats (One way ANOVA: $F(2,43)=2.207, P=0.1223$ ).

(E) Quantification of percentage area of fluorescence of IOFC GFAP from 3-4 slices averaged by animal. GFAP staining in IOFC of extended access rats $(n=5)$ is greater than that of that 
from chow $\left(n=5, P<0.01^{\# \#}\right)$ or restricted access rats $\left(n=4, P<0.05^{\#}\right)$ One way ANOVA: $F(2,11)$ $=7.526, \mathrm{P} 0.0087)$.

(F) Quantification of relative intensity units of IOFC GFAP fluorescence from 3-4 slices averaged by animal. GFAP staining in IOFC of extended access rats $(n=5)$ is greater than that of that from chow $\left(n=5, P<0.01^{\# \#}\right)$ or restricted access rats $\left(n=4, P<0.05^{\#}\right)$, One way ANOVA: $F(2,11)$ $=12.08, \mathrm{P}=0.0017)$.

(G) Quantification of the number of GFAP expressing cells from 3-4 slices averaged by animal. There is no significant difference in the number of GFAP+ cells in IOFC of chow $(n=5)$, restricted $(n=4)$ or extended access rats $(n=5$; One way ANOVA: $F(2,11)=0.314, P=0.737)$. (H) Representative images illustrating fluorescent dye-filling of astrocytes within layer II/III of the IOFC in slices from chow and extended access rats. Scale bars: $10 \mu \mathrm{m}$. Inset, experimental schematic outlining imaging of astrocyte processes. Coronal slices containing OFC were first loaded with the astrocyte-specific dye, sulfurhodamine 101 (SR101 $20 \mu \mathrm{M}$ ) for 20 mins. SR101 labelled astrocytes were then patch-clamped under fluorescence, filled with the dye, FITCdextran to label the fine processes and then imaged using 2-photon microscopy.

(I) Area of individual astrocyte territory in IOFC from chow $(n=11 / 4)$ and extended $(n=15 / 4)$ access rats. Astrocyte territory is significantly increased in IOFC from extended access rats compared to chow access rats $\left(\mathrm{t}_{(24)}=2.514, \mathrm{P}=0.019^{\#}\right)$.

(J) Area of individual astrocyte territory normalized to averaged chow territory. Normalized astrocyte territory is significantly increased in IOFC from extended access rats $(n=15 / 4)$ compared to chow access rats $(n=11 / 4)\left(t_{(24)}=2.514, P=0.019^{\#}\right)$. (K) Astrocyte process arborization indicated by the Schoenen ramification index. Astrocyte ramification is not different between chow $(n=10 / 4)$ and extended access $(14 / 4)$ rats $\left(t_{(22)}=\right.$ 0.532, $P=0.6003)$. Two cells were omitted due to high background fluorescence.

Bars represent mean \pm s.e. $m$ with individual values overlaid. 


\section{Figure 7: Astrocytes within the IOFC of rats with extended access to an obesogenic diet}

\section{have impaired glutamate transport.}

(A) Experimental schematic illustrating the recording of glutamate transporter currents in astrocytes. SR-101 labelled astrocytes were patched-clamped at $-80 \mathrm{mV}$ with a caged RubiGlutamate in the extracellular ACSF. Glutamate was focally activated on the astrocyte via a blue LED $(470 \mathrm{~nm})$ light flash. A glutamate transporter current was isolated in the presence of synaptic blockers.

(B) Example traces showing glial glutamate transporter currents evoked via flash photolysis $(470 \mathrm{~nm})$ of RuBi-Glutamate $(30 \mu \mathrm{M})$ before and during application of the glutamate transport blocker, TBOA $(30 \mu \mathrm{M})$, in the IOFC of chow and extended access rats. Scale bar: $10 \mathrm{pA}, 25$ ms.

(C) Bar graph quantifying the amplitude of flash-evoked GLT-1 currents in astrocytes. GLT-1 current amplitude is significantly reduced in IOFC astrocytes from extended $(n=7 / 4)$ compared to chow $(n=15 / 5)$ access rats $\left(t_{(20)}=3.664, P=0.0015^{\# \#}\right)$.

(D) Example immunoblots of GLT-1 from the OFC of chow or extended access rats. Beta-actin is the loading control protein. (Exposure 600s for GLT-1 and 95s for beta-actin).

(E) Western blot analyses of total GLT-1 levels in the OFC of chow ( $N=8$ rats) or extended $(\mathrm{N}=$ 9 rats) access rats. There is no difference in GLT-1 expression between Chow and Extended access rats $\left(t_{(15)}=0.159, P=0.88\right)$.

(F) Experimental schematic illustrating the effect of TBOA on evoked IPSCs.

(G) Blockade of glutamate transport with TBOA (30 $\mu \mathrm{M})$ induces a long-term depression of IPSCs of IOFC pyramidal neurons from chow access rats $(n=19 / 11)$. This effect is inhibited when slices are pre-treated with the CB1 receptor antagonist, AM251 (3 $\mu \mathrm{M}, \mathrm{n}=7 / 4)$. This 
TBOA-induced LTD is occluded in IOFC neurons of extended access rats $(n=8 / 5)$. Mixed effects model $(R E M L)$ : Diet $x$ time interaction: $F(78,1006)=8.556, P<0.0001$, diet effect: $F(2,31)=27.21, P<0.0001$. Dotted vertical lines indicate the time period analyzed in $(H)$. (H) Bar graph quantifying the percentage change of evoked IPSC amplitude during TBOA application in slices from untreated $(n=11 / 8)$ and AM251-treated $(3 \mu M, n=6 / 4)$ neurons from chow and extended $(\mathrm{n} / \mathrm{N}=8 / 5)$ access rats. TBOA produces a long-term suppression of evoked IPSC amplitude of IOFC neurons from chow access rats, which is blocked in the presence of AM251 and absent in extended access rats. One way ANOVA: $F(2,22)=11.81, P=0.0003$, Tukey's multiple comparison test: chow vs. chow $+\mathrm{AM} 251, \mathrm{P}<0.001^{\# \# \#}$, chow vs. extended, $\mathrm{P}<0.05^{\#}$. Evoked IPSCs after TBOA is significantly decreased from baseline in IOFC neurons from chow $\left(t(10)=6.016, P=0.0001^{\star * *}\right)$, but not in the presence of $A M 251(t(5)=1.845, P=$ $0.124)$ nor in extended access rats $(t(7)=0.075, P=0.942)$. Cells that died before the analysis were excluded from the bar graph.

Bars represent mean \pm s.e. $m$ with individual values overlaid.

Figure 8: Restoration of glutamate homeostasis reverses the diet-induced alterations in inhibitory synaptic plasticity.

(A) NAC treatment enhances GLT-1 current amplitude in astrocytes from extended access rats (Extended: $\mathrm{n}=8 / 4$, Extended $\left.+\mathrm{NAC}: \mathrm{n}=12 / 3, \mathrm{t}_{(18)}=2.114, \mathrm{P}=0.049^{\#}\right)$. Inset: Example traces of GLT-1 currents from IOFC astrocytes in untreated and NAC-treated slices from extended access rats. Scale bars: 10pA, $25 \mathrm{~ms}$.

(B) Upper, NAC treatment reduces baseline NMDA EPSC half-decay time in IOFC pyramidal neurons of extended access rats (Extended: $n=8 / 2$, Extended $+N A C: n=10 / 4, t_{(16)}=2.148, P$ $\left.=0.047^{\#}\right)$. Lower, change in decay time of evoked NMDA EPSCs in response to application of the glutamate transporter inhibitor, TBOA (10 min, $30 \mu \mathrm{M})$. Decay time is enhanced by TBOA in 
IOFC neurons of both extended access $\left(n=8 / 2, t_{(7)}=10.01, P<0.0001^{* \star \star *}\right)$ and NAC treated slices from extended access rats compared to baseline $\left(n=9 / 4, t_{(8)}=7.204, P<0.0001^{\star \star \star \star}\right)$. NAC treatment restores TBOA's capacity to enhance this measure $\left(t_{(15)}=3.119, P=0.007^{\# \#}\right)$. Inset: Example traces of evoked NMDA EPSCs normalized to peak amplitude from IOFC pyramidal neurons in response to $5 \mathrm{X} 100 \mathrm{~Hz}$ stimulation in untreated and NAC-treated slices from rats with extended access to an obesogenic diet. Artefacts minimized for clarity. Scale bars: $0.5,50 \mathrm{~ms}$.

(C) NAC treatment $(n=8 / 6)$ abolishes the facilitation of evoked IPSC amplitude unmasked by the neutral CB1 receptor antagonist, NESS-0327 (0.5 $\mu \mathrm{M})$ application to IOFC slices of extended access rats $(n=9 / 7)$. A mixed-effects model (REML) indicates a significant NAC treatment $x$ time interaction $(F(29,427)=2.406, p<0.0001)$ and a main effect of NAC treatment $(F(1,15)=10.69, P=0.0052)$. Broken vertical lines indicate time period analyzed in $(D)$. (D) Bar graph quantifying evoked IPSC amplitude 15-20 min during NESS-0327 (0.5 $\mu \mathrm{M})$ application in untreated vs. NAC treated rats. There was a significant increase of IPSC amplitude over baseline with NESS-0327 treatment in IOFC neurons of extended access rats $\left(t_{(8)}=3.351, P=0.0101^{*}\right)$, but not when slices were treated with $N A C\left(t_{(7)}=0.338, P=0.745\right)$. There was a significant difference between IOFC neurons from extended access rats treated with NAC and those untreated (Extended: $n=9 / 7$, Extended + NAC: $n=8 / 6, t_{(15)}=2.623, P=0.019^{\#}$ ).

(E) NAC treatment restores the ability to induce mGluR LTD with the mGluR1/5 agonist, DHPG in IOFC neurons of extended access rats $(n=11 / 8)$.

(F) Time plot of evoked IPSC amplitude before and during TBS in IOFC neurons pretreated with NAC of extended $(n=14 / 7)$ access rats. TBS-LTD was not present in IOFC neurons of extended access rats treated with the selective GLT-1 inhibitor, DHK ( $n=16 / 12)$. Dotted vertical lines indicate the 25-30 min time period following TBS analyzed in (G). There was a significant 
interaction of drug group $x$ timecourse $(F(48,1270)=5.302, P<0.0001)$ and a main effect of $\operatorname{drug}\left(F(1,28)=20.96, P<0.0001^{* \star * *}(\right.$ Mixed-effect model 2-way RM ANOVA).

(G) Bar graph quantifying evoked IPSC amplitude in NAC treated slices after TBS in the presence or absence $(n=14 / 7)$ of DHK $(n=16 / 12)$ or after DHPG $(n=11 / 3)$. NAC treatment restores the ability to induce TBS- $\left(t_{(13)}=4.564, P=0.0005^{\star \star *}\right.$, one-sample t-test difference from baseline) or mGluR- $\left(t_{(10)}=5.303, P=0.0003^{\star \star *}\right.$, one-sample t-test difference from baseline) LTD in IOFC neurons of extended access rats. This rescue of TBS LTD was prevented in the presence of $\mathrm{DHK}\left(\mathrm{t}_{(15)}=0.673, \mathrm{P}=0.5024\right.$, one-sample $\mathrm{t}$-test compared to baseline $)$. There was a significant difference between groups (One way ANOVA $F(2,38)=9.992, P=0.003$, Tukey's posthoc test: NAC-TBS vs NAC-TBS+DHK, P<0.01"\#).

(H) Effect of NAC on mIPSCs rate onto IOFC pyramidal neurons from chow or extended access rats (2-way ANOVA: diet $x$ drug interaction: $F(1,91)=18.53, P<0.0001)$, diet effect: $F(1,91=$ 13.67, $P=0.0004$, drug effect: $F(1,91)=22.03, P<0.0001)$. A Tukey's posthoc test indicates that NAC treatment enhances mIPSC rate of IOFC neurons from extended access rats

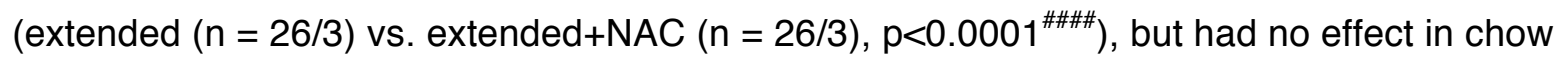
access rats (chow $(n=20 / 3)$ vs. chow+NAC $(n=24 / 3), P>0.05)$. Further, mIPSC rate was

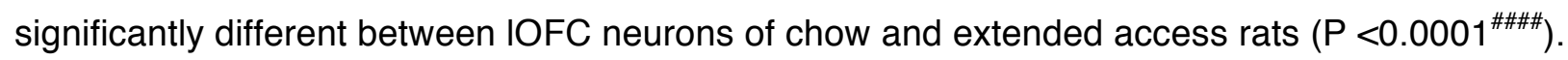
Inset: Example traces of mIPSCs in untreated and NAC-treated slices from extended access rats. Scale bar: $20 \mathrm{pA}, 100 \mathrm{~ms}$.

(I) Effect of NAC on mIPSCs amplitude of IOFC pyramidal neurons from chow or extended access rats (2-way ANOVA: diet $x$ drug interaction: $F(1,91)=12.45, P=0.0007)$, diet effect: $F(1,91=0.0006, P=0.9803$, drug effect: $F(1,91)=13.47, P=0.0004)$. A Tukey's posthoc test indicates that NAC did not alter mIPSC amplitude of IOFC neurons from chow access rats ( $p>0.05$ ), but enhanced this measure in IOFC neurons from extended access rats

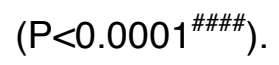


bioRxiv preprint doi: https://doi.org/10.1101/2020.05.01.073205; this version posted May 3, 2020. The copyright holder for this preprint (which was not certified by peer review) is the author/funder, who has granted bioRxiv a license to display the preprint in perpetuity. It is made available under aCC-BY-NC-ND 4.0 International license.

Bars represent mean \pm s.e.m with individual values overlaid. 
Figure S1. GABAergic synapses onto IOFC pyramidal neurons exhibit endocannabinoidinduced long-term depression.

(A) Schematic showing TBS in the presence or absence of the CB1 receptor antagonist, AM251 or the mGluR5 antagonist, MTEP onto IOFC pyramidal neurons.

(B) Example traces of evoked IPSCs before (black) and after (grey) TBS-induced LTD in control, AM251 $(3 \mu \mathrm{M})$ or MTEP $(10 \mu \mathrm{M})$ onto IOFC neurons of naïve rats. Scale bars: $250 \mathrm{pA}, 25 \mathrm{~ms}$.

(C) Time plot of evoked IPSC amplitude before and after TBS in IOFC neurons of naïve rats $(\mathrm{n}=$ 8/4). TBS was blocked by AM251 (3 $\mu \mathrm{M}, \mathrm{n}=17 / 5)$ or MTEP $(10 \mu \mathrm{M}, \mathrm{n}=8 / 4)$. Dotted vertical lines indicate the 25-30 min time period following TBS analyzed in (C). There was a significant interaction of drug group $x$ timecourse $(F(96,1174)=4.752, P<0.0001)$ and a main effect of $\operatorname{drug}\left(\mathrm{F}(2,28)=35.48, \mathrm{P}<0.0001^{\star * \star *}\right.$ (Mixed effects model 2-way RM ANOVA).

(D) Bar graph quantifying the percentage change in evoked IPSC amplitude 25-30 mins following TBS in IOFC neurons from naive rats. TBS-LTD is present in IOFC neurons $(n=8 / 4)$, but not AM251- $(n=11 / 5)$ or MTEP-treated IOFC neurons $(n=7 / 4)$, One-way ANOVA: $(F(2,23)=31.92, P<0.0001)$, Tukey's multiple comparison test control vs $A M 251, P<0.0001^{\# \# \#, ~}$ control vs MTEP, $\mathrm{P}<0.0001^{\# \# \# \#}$, AM251 vs MTEP, P $>0.05$. Evoked IPSCs after TBS is significantly decreased from baseline in IOFC neurons from control $\left(t_{(7)}=7.186, P=0.0002^{\star \star \star}\right)$, AM251-treated $\left(t_{(10)}=3.139, P=0.0105^{\star *}\right)$, but not MTEP-treated neurons $\left(t_{(6)}=0.7110\right.$, $\mathrm{P}=0.504)$.

(E) Bar graph of the paired-pulse ratio of evoked IPSC amplitude before (Baseline) and after TBS (Post-TBS) in control, AM251-treated, or MTEP-treated IOFC neurons. A RM 2-way ANOVA gives a significant drug $x \operatorname{LTD}$ interaction $(F(2,20)=5.804, P=0.0103)$. A Sidak's multiple comparison test reveals a significant effect of TBS on paired-pulse facilitation in control IOFC neurons $\left(n=8 / 4, P<0.05^{\#}\right)$, but not AM251 $(n=8 / 5, P>0.05)$ or MTEP-treated neurons $(n=6 / 4, P>0.05)$. 
Bars represent mean \pm s.e.m with individual values overlaid.

\section{Graphical Summary. Summary schematic of obesity-induced synaptic alterations in the}

IOFC. Endocannabinoids mediate mGluR-dependent LTD at inhibitory synapses onto layer II/III

pyramidal neurons of the IOFC. Endocannabinoid-mediated plasticity is impaired following an obesogenic diet. This is mediated upstream by extrasynaptic glutamate activation of group 1 mGluRs due to impairment of astrocytic GLT-1 function. N-acetylcysteine restores glutamate homeostasis and restores endocannabinoid function in obese rats. 


\section{REFERENCES}

Baker, D.A., McFarland, K., Lake, R.W., Shen, H., Tang, X.C., Toda, S., and Kalivas, P.W. (2003). Neuroadaptations in cystine-glutamate exchange underlie cocaine relapse. Nature Neuroscience.

Brown, R.M., Kupchik, Y.M., and Kalivas, P.W. (2013). The story of glutamate in drug addiction and of $\mathrm{N}$-acetylcysteine as a potential pharmacotherapy. JAMA Psychiatry.

Bushong, E.A., Martone, M.E., Jones, Y.Z., and Ellisman, M.H. (2002). Protoplasmic astrocytes in CA1 stratum radiatum occupy separate anatomical domains. J. Neurosci. 22, 183-192.

Cano, V., Valladolid-Acebes, I., Hernández-Nuño, F., Merino, B., Olmo, N. Del, Chowen, J.A., and Ruiz-Gayo, M. (2014). Morphological changes in glial fibrillary acidic protein immunopositive astrocytes in the hippocampus of dietary-induced obese mice. NeuroReport.

Chen, R., Zhang, J., Fan, N., Teng, Z.Q., Wu, Y., Yang, H., Tang, Y.P., Sun, H., Song, Y., and Chen, C. (2013). X $\Delta$ 9-THC-caused synaptic and memory impairments are mediated through COX-2 signaling. Cell.

Chevaleyre, V., and Castillo, P.E. (2004). Endocannabinoid-mediated metaplasticity in the hippocampus. Neuron 43, 871-881.

Danbolt, N.C., Storm-Mathisen, J., and Kanner, B.I. (1992). An [Na++ K+]coupled L-glutamate transporter purified from rat brain is located in glial cell processes. Neuroscience 51, 295-310.

Dludla, P.V., Mazibuko-Mbeje, S.E., Nyambuya, T.M., Mxinwa, V., Tiano, L., Marcheggiani, F., Cirilli, I., Louw, J., and Nkambule, B.B. (2019). The beneficial effects of N-acetyl cysteine (NAC) against obesity associated complications: A systematic review of pre-clinical studies. Pharmacol. Res. 146, 104332.

Drew, G.M., Mitchell, V.A., and Vaughan, C.W. (2008). Glutamate spillover modulates GABAergic synaptic transmission in the rat midbrain periaqueductal grey via metabotropic glutamate receptors and endocannabinoid signaling. Journal of Neuroscience.

Gatta-Cherifi, B., and Cota, D. (2016). New insights on the role of the endocannabinoid system in the regulation of energy balance. International Journal of Obesity 26, 114-124.

Gómez-Pinilla, F. (2008). Brain foods: The effects of nutrients on brain function. Nature Reviews Neuroscience.

Grant, J.E., Odlaug, B.L., and Won Kim, S. (2009). N-Acetylcysteine, a Glutamate Modulator, in the Treatment of Trichotillomania. Archives of General Psychiatry.

Grant, J.E., Chamberlain, S.R., Redden, S.A., Leppink, E.W., Odlaug, B.L., and Kim, S.W. (2016). N -Acetylcysteine in the Treatment of Excoriation Disorder. JAMA Psychiatry. 
Gremel, C.M., and Costa, R.M. (2013). Orbitofrontal and striatal circuits dynamically encode the shift between goal-directed and habitual actions. Nat Commun 4, 2264.

Gremel, C.M., Chancey, J.H., Atwood, B.K., Luo, G., Neve, R., Ramakrishnan, C., Deisseroth, K., Lovinger, D.M., and Costa, R.M. (2016). Endocannabinoid Modulation of Orbitostriatal Circuits Gates Habit Formation. Neuron 90, 1312-1324.

Hall, K.D., and Guo, J. (2017). Obesity Energetics: Body Weight Regulation and the Effects of Diet Composition. Gastroenterology 152, 1718-1727.e3.

Jennings, J.H., Kim, C.K., Marshel, J.H., Raffiee, M., Ye, L., Quirin, S., Pak, S., Ramakrishnan, C., and Deisseroth, K. (2019). Interacting neural ensembles in orbitofrontal cortex for social and feeding behaviour. Nature.

Jiang, B., Huang, S., de Pasquale, R., Millman, D., Song, L., Lee, H.-K., Tsumoto, T., and Kirkwood, A. (2010). The maturation of GABAergic transmission in visual cortex requires endocannabinoidmediated LTD of inhibitory inputs during a critical period. Neuron 66, 248-259.

Johnson, P.M., and Kenny, P.J. (2010). Dopamine D2 receptors in addiction-like reward dysfunction and compulsive eating in obese rats. Nat. Neurosci. 13, 635-641.

Kirkham, T.C., Williams, C.M., Fezza, F., and Di Marzo, V. (2002). Endocannabinoid levels in rat limbic forebrain and hypothalamus in relation to fasting, feeding and satiation: stimulation of eating by 2-arachidonoyl glycerol. British Journal of Pharmacology 136, 550-557.

Kupchik, Y.M., Moussawi, K., Tang, X.-C., Wang, X., Kalivas, B.C., Kolokithas, R., Ogburn, K.B., and Kalivas, P.W. (2012). The effect of $\mathrm{N}$-acetylcysteine in the nucleus accumbens on neurotransmission and relapse to cocaine. Biol. Psychiatry 71, 978-986.

Lafourcade, M., Larrieu, T., Mato, S., Duffaud, A., Sepers, M., Matias, I., De Smedt-Peyrusse, V., Labrousse, V.F., Bretillon, L., Matute, C., et al. (2011). Nutritional omega-3 deficiency abolishes endocannabinoid-mediated neuronal functions. Nature Neuroscience 14, 345-350.

Lau, B.K., Cota, D., Cristino, L., and Borgland, S.L. (2017). Endocannabinoid modulation of homeostatic and non-homeostatic feeding circuits. Neuropharmacology.

Linehan, V., Fang, L.Z., and Hirasawa, M. (2018). Short-term high-fat diet primes excitatory synapses for long-term depression in orexin neurons. Journal of Physiology.

Lowe, C.J., Reichelt, A.C., and Hall, P.A. (2019). The Prefrontal Cortex and Obesity: A Health Neuroscience Perspective. Trends Cogn. Sci. (Regul. Ed.) 23, 349-361.

Manduca, A., Bara, A., Larrieu, T., Lassalle, O., Joffre, C., Layé, S., and Manzoni, O.J. (2017). Amplification of mGlu5-endocannabinoid signaling rescues behavioral and synaptic deficits in a mouse model of adolescent and adult dietary polyunsaturated fatty acid imbalance. Journal of Neuroscience. 
Di Marzo, V. (2011). Endocannabinoids: an appetite for fat. Proceedings of the National Academy of Sciences of the United States of America 108, 12567-12568.

Di Marzo, V., and Matias, I. (2005). Endocannabinoid control of food intake and energy balance. Nature Neuroscience.

Matikainen-Ankney, B.A., and Kravitz, A.V. (2018). Persistent effects of obesity: a neuroplasticity hypothesis. Ann. N. Y. Acad. Sci. 1428, 221-239.

McBean, G.J. (2002). Cerebral cystine uptake: a tale of two transporters. Trends Pharmacol. Sci. 23, 299-302.

Moussawi, K., Pacchioni, A., Moran, M., Olive, M.F., Gass, J.T., Lavin, A., and Kalivas, P.W. (2009). N-Acetylcysteine reverses cocaine-induced metaplasticity. Nature Neuroscience.

Murphy-Royal, C., Dupuis, J.P., Varela, J.A., Panatier, A., Pinson, B., Baufreton, J., Groc, L., and Oliet, S.H.R. (2015). Surface diffusion of astrocytic glutamate transporters shapes synaptic transmission. Nat. Neurosci. 18, 219-226.

Oliet, S.H.R., Piet, R., and Poulain, D.A. (2001). Control of glutamate clearance and synaptic efficacy by glial coverage of neurons. Science.

Ongür, D., and Price, J.L. (2000). The organization of networks within the orbital and medial prefrontal cortex of rats, monkeys and humans. Cereb. Cortex 10, 206-219.

Papouin, T., Dunphy, J., Tolman, M., Foley, J.C., and Haydon, P.G. (2017). Astrocytic control of synaptic function. Philos. Trans. R. Soc. Lond., B, Biol. Sci. 372.

Ravinet Trillou, C., Delgorge, C., Menet, C., Arnone, M., and Soubrié, P. (2004). CB1 cannabinoid receptor knockout in mice leads to leanness, resistance to diet-induced obesity and enhanced leptin sensitivity. International Journal of Obesity $28,640-648$.

Reissner, K.J., Gipson, C.D., Tran, P.K., Knackstedt, L.A., Scofield, M.D., and Kalivas, P.W. (2015). Glutamate transporter GLT-1 mediates N-acetylcysteine inhibition of cocaine reinstatement. Addiction Biology.

Rolls, E.T. (2004). Convergence of sensory systems in the orbitofrontal cortex in primates and brain design for emotion. In Anatomical Record - Part A Discoveries in Molecular, Cellular, and Evolutionary Biology, p.

Rolls, B.J., Rowe, E.A., and Turner, R.C. (1980). Persistent obesity in rats following a period of consumption of a mixed, high energy diet. J. Physiol. (Lond.) 298, 415-427.

Rothstein, J.D., Martin, L., Levey, A.I., Dykes-Hoberg, M., Jin, L., Wu, D., Nash, N., and Kuncl, R.W. (1994). Localization of neuronal and glial glutamate transporters. Neuron 13, 713-725. 
Rubino, T., Realini, N., Braida, D., Guidi, S., Capurro, V., Viganò, D., Guidali, C., Pinter, M., Sala, M., Bartesaghi, R., et al. (2009). Changes in hippocampal morphology and neuroplasticity induced by adolescent THC treatment are associated with cognitive impairment in adulthood. Hippocampus.

Seabrook, L.T., and Borgland, S.L. (2020). The orbitofrontal cortex, food intake and obesity. J Psychiatry Neurosci 45, 190163.

Shimamoto, K., Shigeri, Y., Yasuda-Kamatani, Y., Lebrun, B., Yumoto, N., and Nakajima, T. (2000). Syntheses of optically pure $\beta$-hydroxyaspartate derivatives as glutamate transporter blockers. Bioorganic and Medicinal Chemistry Letters.

Simopoulos, A.P. (2016). An increase in the Omega-6/Omega-3 fatty acid ratio increases the risk for obesity. Nutrients 8 .

Soria-Gómez, E., Bellocchio, L., Reguero, L., Lepousez, G., Martin, C., Bendahmane, M., Ruehle, S., Remmers, F., Desprez, T., Matias, I., et al. (2014). The endocannabinoid system controls food intake via olfactory processes. Nature Neuroscience 17, 407-415.

Spencer, S.J., Korosi, A., Layé, S., Shukitt-Hale, B., and Barrientos, R.M. (2017). Food for thought: how nutrition impacts cognition and emotion. Npj Science of Food.

Spiegelman, B.M., and Flier, J.S. (2001). Obesity and the regulation of energy balance. Cell.

Thaler, J.P., Yi, C.-X., Schur, E.A., Guyenet, S.J., Hwang, B.H., Dietrich, M.O., Zhao, X., Sarruf, D.A., Izgur, V., Maravilla, K.R., et al. (2012). Obesity is associated with hypothalamic injury in rodents and humans. J. Clin. Invest. 122, 153-162.

Thoeni, S., Loureiro, M., O’Connor, E.C., and Lüscher, C. (2020). Depression of Accumbal to Lateral Hypothalamic Synapses Gates Overeating. Neuron.

Thomazeau, A., Bosch-Bouju, C., Manzoni, O., and Laye, S. (2016). Nutritional n-3 PUFA Deficiency Abolishes Endocannabinoid Gating of Hippocampal Long-Term Potentiation. Cerebral Cortex (New York, N.Y. : 1991).

Thompson, J.L., Drysdale, M., Baimel, C., Kaur, M., MacGowan, T., Pitman, K.A., and Borgland, S.L. (2017). Obesity-Induced Structural and Neuronal Plasticity in the Lateral Orbitofrontal Cortex. Neuropsychopharmacology 1-11.

Tsai, S.F., Chen, Y.W., and Kuo, Y.M. (2018). High-fat diet reduces the hippocampal content level of lactate which is correlated with the expression of glial glutamate transporters. Neuroscience Letters.

Valladolid-Acebes, I., Merino, B., Principato, A., Fole, A., Barbas, C., Lorenzo, M.P., García, A., del Olmo, N., Ruiz-Gayo, M., and Cano, V. (2012). High-fat diets induce changes in hippocampal 
glutamate metabolism and neurotransmission. American Journal of Physiology - Endocrinology and Metabolism.

Zhang, Y., Reichel, J.M., Han, C., Zuniga-Hertz, J.P., and Cai, D. (2017). Astrocytic Process Plasticity and IKK $\beta /$ NF-KB in Central Control of Blood Glucose, Blood Pressure, and Body Weight. Cell Metabolism.

Zhao, L., Yeh, M.L.-W., and Levine, E.S. (2015). Role for Endogenous BDNF in EndocannabinoidMediated Long-Term Depression at Neocortical Inhibitory Synapses. ENeuro 2. 


\section{StAR Methods:}

Subjects: All experiments on rats were approved by the Animal Care Committee of the University of Calgary, under guidelines set by the Canadian Council on Animal Care. Male Long-Evans rats (P50-55) were obtained from Charles River Laboratories and were individually housed in cages with paper bedding (Alphapads, Lawrenceville, GA) on a 12:12 reverse light dark cycle (lights on at 8:00 am).

Diets: In addition to ad-libitum chow and water, rats were given access to a cafeteria diet (Kraft $^{\mathrm{TM}}$ smooth peanut butter, chocolate Timbit $^{\mathrm{TM}}$ donut holes, Kirkland ${ }^{\mathrm{TM}}$ beef hotdogs, Froot Loops $^{\mathrm{TM}}$, Doritos ${ }^{\mathrm{TM}}$ ) for either $24 \mathrm{~h}$ per day (Extended access), $1 \mathrm{~h}$ per day (Restricted access) or $0 \mathrm{~h}$ per day (Chow only). Energy density and macronutrient composition is listed in supplemental Table 1. Rats with restricted access to the cafeteria diet were given their $1 \mathrm{~h}$ access to cafeteria diet approximately $2 \mathrm{~h}$ into their dark cycle. Rats' body weights were measured immediately before and after 40 days of exposure to the cafeteria diet.

Slice preparation: Rats were deeply anaesthetized with isoflurane and intracardially perfused with N-methyl D-glucamine (NMDG) solution of composition (in mM): 93 NMDG, $2.5 \mathrm{KCl}, 1.2$ $\mathrm{NaH}_{2} \mathrm{PO}_{4} \cdot \mathrm{H}_{2} \mathrm{O}, 30 \mathrm{NaHCO}_{3}, 20$ HEPES, 25 D-glucose, 5 sodium ascorbate, 3 sodium pyruvate, 2 thiourea, $10 \mathrm{MgSO}_{4} \cdot 7 \mathrm{H}_{2} \mathrm{O}, 0.5 \mathrm{CaCl}_{2} .2 \mathrm{H}_{2} \mathrm{O}$. Rats were then decapitated and coronal sections $(300 \mu \mathrm{m})$ containing orbitofrontal cortex (OFC) were cut in NMDG solution using a vibratome (VT1200, Leica Microsystems, Nussloch, Germany). Slices were recovered in warm NMDG solution $\left(32^{\circ} \mathrm{C}\right.$ ) for $10-12$ min before being transferred to a holding chamber containing artificial cerebrospinal fluid (ACSF) of composition (in mM): $126 \mathrm{NaCl}, 1.6 \mathrm{KCl}, 1.1 \mathrm{NaH}_{2} \mathrm{PO}_{4}, 1.4 \mathrm{MgCl}_{2}$, 2.4 $\mathrm{CaCl}_{2}, 26 \mathrm{NaHCO}_{3}, 11$ glucose $\left(32{ }^{\circ} \mathrm{C}\right)$; equilibrated with 95\% O2 / 5\% $\mathrm{CO}_{2}$. 
Neuronal Electrophysiology: Slices were transferred to a chamber on an upright microscope (Olympus BX51WI) and continuously superfused with ACSF (2 mL. $\left.\mathrm{min}^{-1}, 34{ }^{\circ} \mathrm{C}\right)$. OFC neurons were visualized with a 40X water immersion objective using Dodt gradient contrast optics. Whole-cell voltage-clamp recordings (holding potential $=-70 \mathrm{mV}$ ) of synaptic currents were made using a MultiClamp 700B amplifier (Axon Instruments, Molecular Devices). Pyramidal neurons in layer II/II of the lateral OFC $(\sim 100-300 \mu \mathrm{m}$ above the inflection point of the rhinal sulcus) were identified by morphological and electrophysiological characteristics (triangular shape, capacitance $>100 \mathrm{pF}$, input resistance $<100 \mathrm{mOhm}$ ). For recording of inhibitory postsynaptic currents (IPSCs), recordings were obtained with an internal solution of the following composition (in mM): $80 \mathrm{CsCH}_{3} \mathrm{SO}_{3}, 60 \mathrm{CsCl}, 10 \mathrm{HEPES}, 0.2 \mathrm{EGTA}, 1 \mathrm{MgCl}_{2}, 2$ MgATP, 0.3 NaGTP, 5 QX-314-Cl (pH 7.2-7.3, 305 mOsm). Inhibitory postsynaptic currents (IPSCs) were pharmacologically isolated with the a-amino-3-hydroxy-5 methyl-4isoxazolepropionic acid (AMPA)/kainate receptor antagonist, DNQX (10 $\mu \mathrm{M})$ and the glycine receptor antagonist, strychnine $(1 \mu \mathrm{M})$. For recording of excitatory postsynaptic currents (EPSCs), recordings were made using an internal solution containing (in $\mathrm{mM}$ ): $139 \mathrm{CsCH}_{3} \mathrm{SO}_{3}, 8$ CsCl, 10 HEPES, 0.25 EGTA, 4 MgATP, 0.3 NaGTP, 7 Na-phosphocreatine (pH 7.2-7.3, 305 mOsm). EPSCs were pharmacologically isolated with the $\mathrm{GABA}_{\mathrm{A}}$ receptor antagonist, picrotoxin $(100 \mu \mathrm{M})$ and strychnine $(1 \mu \mathrm{M})$. Series resistance $(5-20 \mathrm{M} \Omega)$ and input resistance were monitored online with a $10 \mathrm{mV}$ voltage step given $400 \mathrm{~ms}$ every stimulus. IPSCs and EPSCs were filtered at $2 \mathrm{kHz}$, digitized at $10 \mathrm{kHz}$ and collected using PCLAMP 10 software. In the majority of experiments, electrically-evoked currents were elicited using a bipolar tungsten stimulating electrode (FHC, Maine, USA) placed $\sim 50-300 \mu \mathrm{m}$ laterally from the recorded neuron. Theta-burst stimulation (TBS) consisted of 8 theta-burst trains. Each train contained 10 bursts (200 ms interburst interval), with each burst consisting of 5 stimuli at $100 \mathrm{~Hz}$. Example 
traces of evoked EPSCs or IPSCs were constructed from averages of 30 sweeps. In a subset of experiments, spontaneous miniature EPSCs or mIPSCs were recorded in the presence of the sodium channel blocker, tetrodotoxin (TTX, $0.5 \mu \mathrm{M})$ to inhibit action-potential dependent activity. Individual quantal events were selected based on amplitude $(>10 \mathrm{pA})$, decay time $(<10 \mathrm{~ms})$ and rise time ( $<4 \mathrm{~ms})$ and analyzed using Minianalysis (Synaptosoft).

Astrocyte electrophysiology: Following tissue slicing and recovery, slices were transferred to a temporary holding chamber containing ACSF at physiological temperature and treated with sulforhodamine-101 dye (SR101, $20 \mu \mathrm{M}, 20$ mins) uptake by astrocytes. Slices were then transferred back to a regular ACSF holding solution for at least $30 \mathrm{~min}$. For electrophysiology experiments, SR-101-labelled OFC astrocytes were patch-clamped with a glass electrode $(\sim 4-5$ $\mathrm{m} \Omega$ ) containing internal solution of the following composition (in $\mathrm{mM}$ ): $130 \mathrm{~K}$-Gluconate, $10 \mathrm{KCl}$, 10 HEPES, 0.5 EGTA, 10 Na-phosphocreatine, 4 MgATP, 0.3 NaGTP. In addition to SR-101 labelling, astrocytes were confirmed by their small soma size $(\sim 10 \mu \mathrm{m})$, low resting membrane potential $(<-80 \mathrm{mV})$, low input resistance $(<10 \mathrm{~m} \Omega)$, passive membrane properties (linear current-voltage (IV) relationship) and a lack of action potential firing. Astrocytes were recorded in voltage-clamp configuration $(-80 \mathrm{mV})$ in the presence of the following synaptic blockers: DNQX $(10 \mu \mathrm{M})$, picrotoxin $(100 \mu \mathrm{M})$, strychnine $(1 \mu \mathrm{M})$, the $\mathrm{N}$-methyl-d-aspartate (NMDA) receptor antagonist, D-AP5 $(50 \mu \mathrm{M})$ and the non-selective Group I/II metabotropic glutamate receptor antagonist, MCPG $(250 \mu \mathrm{M})$. Glutamate was applied onto astrocytes by uncaging Rubi-glutamate via flash photolysis (473 nm, $2 \mathrm{~ms}$, Thorlabs). At the conclusion of each recording, the glutamate transport blocker, TBOA $(30 \mu \mathrm{M})$ was finally added to abolish the recorded current. Remaining residual currents were subtracted from the original response to specifically isolate a glutamate transporter current. 
Astrocyte Dye-filling: OFC astrocytes located at a depth of $\sim 20-50 \mu \mathrm{M}$ from the slice surface were patch-clamped with an internal solution of the following composition (in $\mathrm{mM}$ ): $108 \mathrm{~K}$ gluconate, $8 \mathrm{Na}$-gluconate, $2 \mathrm{MgCl}_{2}, 10 \mathrm{HEPES}, 1 \mathrm{~K}_{2}$-EGTA, $4 \mathrm{~K}_{2}-\mathrm{ATP}$, and $0.3 \mathrm{Na}_{3}$-GTP. Once a giga $\Omega$ seal was observed, whole-cell configuration was obtained to initiate filling of astrocytes with Dextran-FITC (3-5 kDa) dye. Access resistance was maintained below $20 \mathrm{M} \Omega$ throughout the experiment. Cells were allowed to fill for a minimum of 10 min before $z$-stack, allowing visualisation of the full morphology of individual astrocytes. Astrocytes were identified by their SR101 dye uptake, presence of vascular endfeet (observed following FITC-dextran loading) and low input resistance (10-20 M 2 ). Fluorescence imaging was performed on a custom two-photon laser-scanning microscope optimized for acute brain slices and patch-clamp electrophysiology. The microscope was equipped with a Ti:Sapph laser (Ultra II, $\sim 4$ W average power, 670-1080 nm, Coherent), objectives (Zeiss 40x NA 1.0, Nikon 16× NA 0.8), a green bandpass emission filter (525-40 nm), an orange/red bandpass emission filter (605-70 nm), and associated photomultiplier tubes (GaAsP Hamamatsu). Z-stacks were used to assess the extent of FITCdextran (3-5kDa) diffusion in individual astrocytes. To image FITC-dextran during astrocyte filling, the Ti:Sapph laser was tuned to $800 \mathrm{~nm}$.

\section{Western Blot Analysis:}

Western blot analysis for GLT-1 was performed on samples of OFC dissected from $100 \mu \mathrm{m}$ thick frozen sections. Samples were homogenized and sonicated in ice-cold RIPA buffer (Thermo Scientific, 89900) with cOmplete Protease Inhibitor Cocktail (Roche. 11836170001) and PhosSTOP (Roche, 4906845001). The homogenate was centrifuged at 13,000 $\mathrm{g}$ for $20 \mathrm{~min}$ at $4^{\circ} \mathrm{C}$. The supernatant was collected and protein content was measured using a Pierce BCA 
Protein Assay Kit (Thermo Scientific, 23225). The samples were denatured with a loading buffer containing 2-mercaptoethanol for 20 minutes at room temperate then the mixture (20 $\mu \mathrm{g} / \mathrm{lane})$ was loaded onto a $10 \%$ sodium dodecyl sulphate-polyacrylamide gel electrophoresis (PAGE) gel and run on a minigel system (Bio-Rad., Mississauga, ON, Canada). After the protein was transferred to PVDF membrane following electrophoresis, the membrane was blocked in $5 \%$ milk followed by the incubation with rabbit anti GLT-1 (Cell Signaling, 3838) at 1:250 at $4^{\circ} \mathrm{C}$ for 24 hours. For loading control, mouse beta-actin (GenScript, A00702) at 1:2500 was applied at room temperature for 1 hour. The blots were further incubated at room temperature for $1 \mathrm{~h}$ with horseradish peroxidase-conjugated goat anti-rabbit IgG 1:2500 for GLT-1 or goat anti-mouse $\operatorname{lgG} 1: 2500$ for beta-actin (Thermo Scientific, 31460 and 31430). Finally, antibody binding was visualized using the enhanced chemiluminescence (ECL) system (Thermo Scientific, 32109) and scanned with a Gel Doc system (Bio-Rad, Mississauga, ON, Canada). The band density of GLT-1 (MW 65 kDa) was analyzed with ImageJ and normalized against the loading control.

\section{Immunohistochemistry:}

Coronal sections containing the OFC were incubated with blocking solution ( $5 \%$ goat serum, $1 \%$ BSA, $0.5 \%$ Triton $\mathrm{X}-100$ in $0.1 \mathrm{M}$ PBS) to prevent nonspecific binding. Floating sections were incubated in primary antibody GFAP (1:1000; MAB360, Millipore) overnight at $4^{\circ} \mathrm{C}$, washed, and then incubated with secondary antibody (goat anti- rabbit, 1: 500) labelled with Alexa Fluor 488 for $1 \mathrm{~h}$ at room temperature. Slices were then mounted on Superfrost Plus clear slides (VWR international), dried, coated with Fluromount medium (Diagnostic Biosystems, Pleasanton, CA, USA) and cover slipped for imaging. Confocal fluorescent images were collected using Nikon Eclipse C1si spectral confocal microscope with motorized stage (Nikon Canada Inc., Ontario, CANADA). The objectives used were 10x PLAN APO DIC (NA 0.45), 20X PLAN APO DIC (NA 
0.75 ) and 60x PLAN APO water immersion DIC (NA 1.20). The laser used was centered at 488 nm (with 515/30 emission filter) wavelengths for detecting GFAP. Threshold levels were adjusted to $20(\mathrm{~min})$ to 225 ( $\max )$ pixels and particle analysis was based on size restriction of 0 $\mathrm{cm}^{2}$ to infinity. All the images were employed the same settings to be consistent for image analysis.

Four coronal slices from the OFC were taken from 5 rats per group. Within each slice, four non-overlapping images per brain region were imaged for a total of 16 images per brain region of a given animal. A total of 80 fields of view were captured per animal at $60 x$ magnification for GFAP staining. All the images were taken using imaging software EZ C1 for Nikon Confocal, Silver Version 3.91 (Nikon, Canada Inc., Ontario, CANADA). Stacked images were acquired using two frames with a resolution of $512 \times 512$ pixels. Offline image processing included maximal intensity projections was conducted using NIS elements. Fluorescence intensity (intensity units, IU) and percentage area of fluorescence was quantified for each image using Image J (1.48v, Wayne Rasband, National Institute of Health, USA). All images were taken blinded to the treatment of the rats.

\section{Data analysis and Statistics}

All statistical analyses were performed in GraphPad Prism 7.02 (GraphPad, US). A two-way repeated measures analysis of variance (RM-ANOVA) was used to examine effects of two factors between multiple groups. With timecourse data, some cells died before the end of recording and therefore matching on every point could not be achieved, therefore we used a mixed-effect model (REML) repeated measures 2-way ANOVA. A one-way ANOVA was used to compare the effect of a treatment between multiple groups. Unless otherwise indicated, a Tukey's multiple comparison test was used to assess within group differences. A two-sample t- 
test was used to compare between two groups when normality distribution was assumed. A one sampled t-test was used to assess difference from $100 \%$. Significance was set at $P<0.05$. A Shapiro-Wilk test was used to assess for normal distribution of data. All data are expressed as mean \pm standard error of the mean (SEM). Individual responses are plotted over averaged responses. Experimental designs and samples sizes aimed at minimizing usage and distress of rats, and were sufficient for detecting robust effect sizes. $\mathrm{N}$ refers to number of rats whereas $\mathrm{n}$ refers to number of cells/number of rats.

\section{Table 1}

Nutrient composition of Cafeteria diet (\% Total diet)

\begin{tabular}{|l|l|l|l|l|}
\hline Food & Fat & Protein & Carbohydrate & Kcal/g \\
\hline Kraft Peanut butter & 53 & 20 & 27 & 6 \\
\hline Kellog's Froot Loops & 3.7 & 3.7 & 89 & 4.1 \\
\hline Doritos & 26 & 6 & 64 & 5.2 \\
\hline Kirkland Hot Dogs & 25 & 12 & 3.5 & 3 \\
\hline $\begin{array}{l}\text { Tim Hortons Timbits } \\
\text { Lab Diet (5P14) Rat } \\
\text { Chow }\end{array}$ & 4.5 & 5.3 & 53 & 3.7 \\
\hline
\end{tabular}




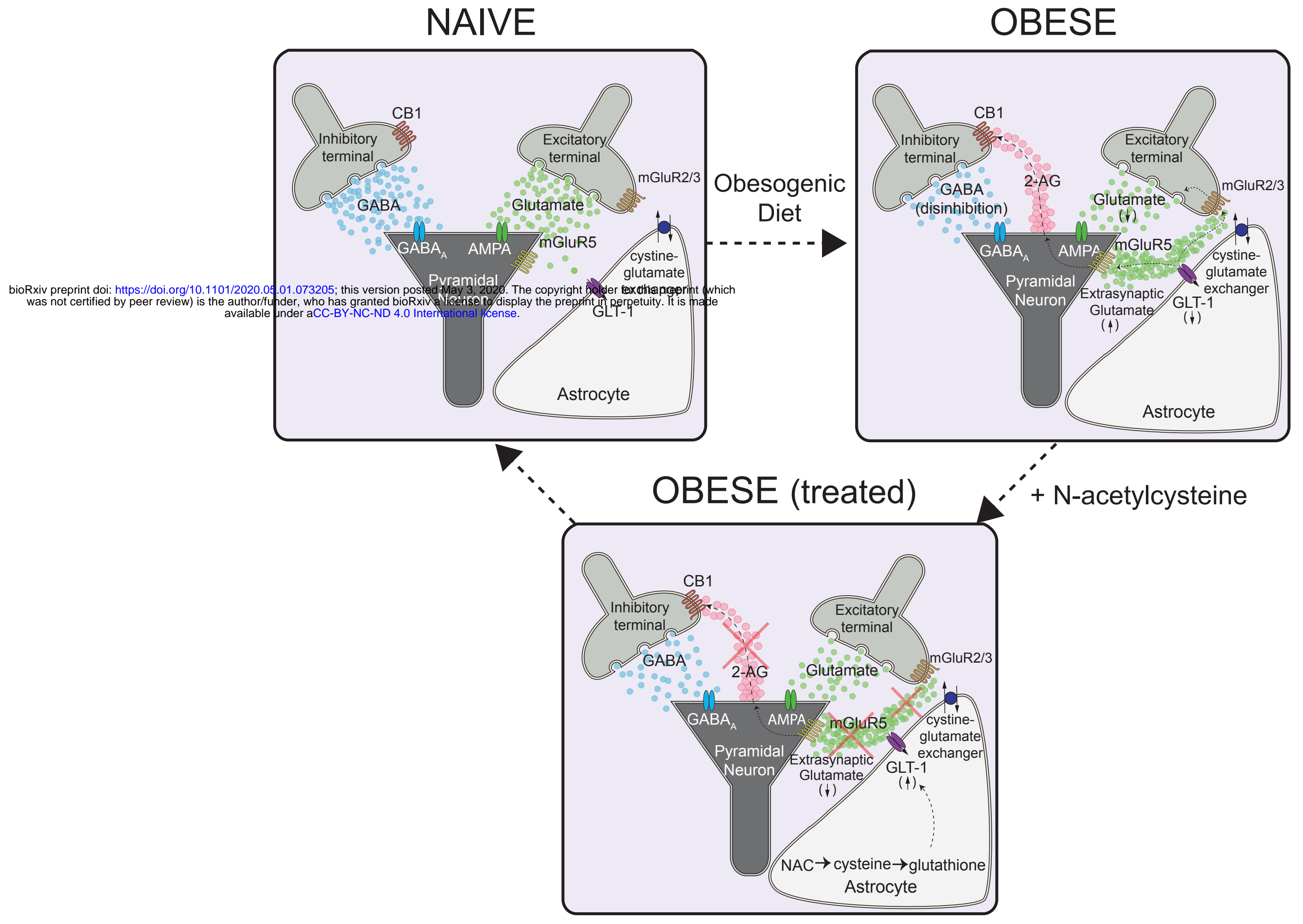


A

\section{OBESOGENIC DIET}

- EXTENDED (24h access)

- RESTRICTED (1h access)

- CHOW (Oh access)
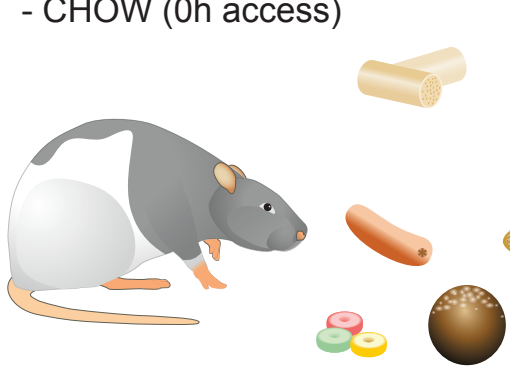

C
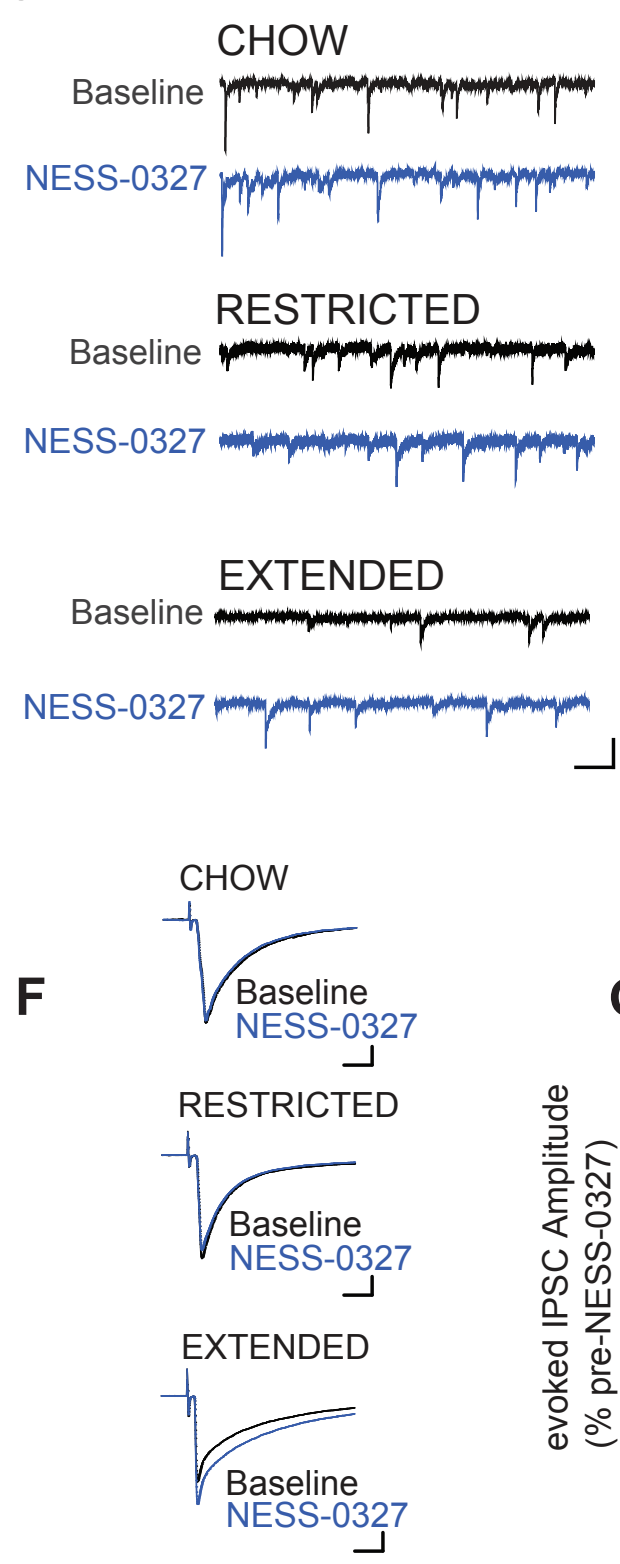
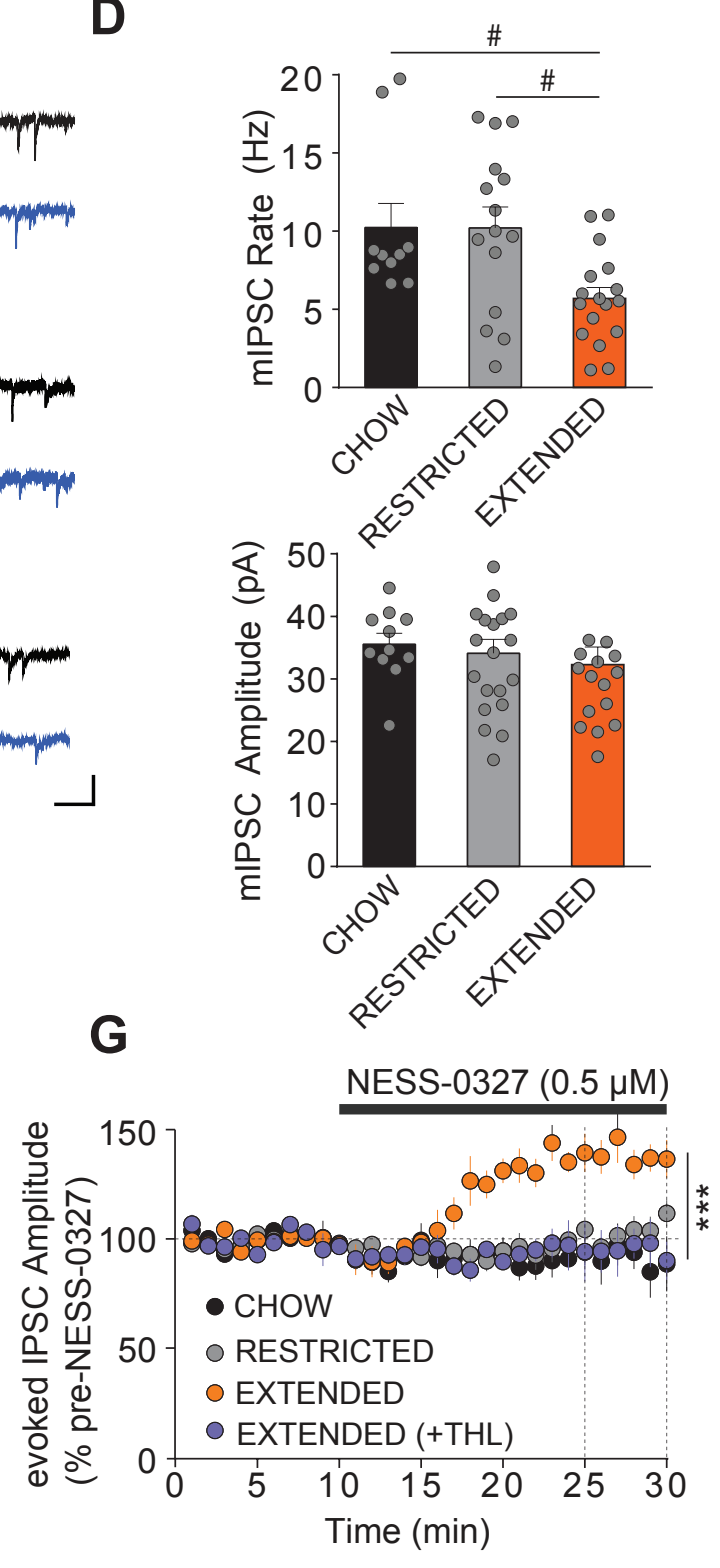

B
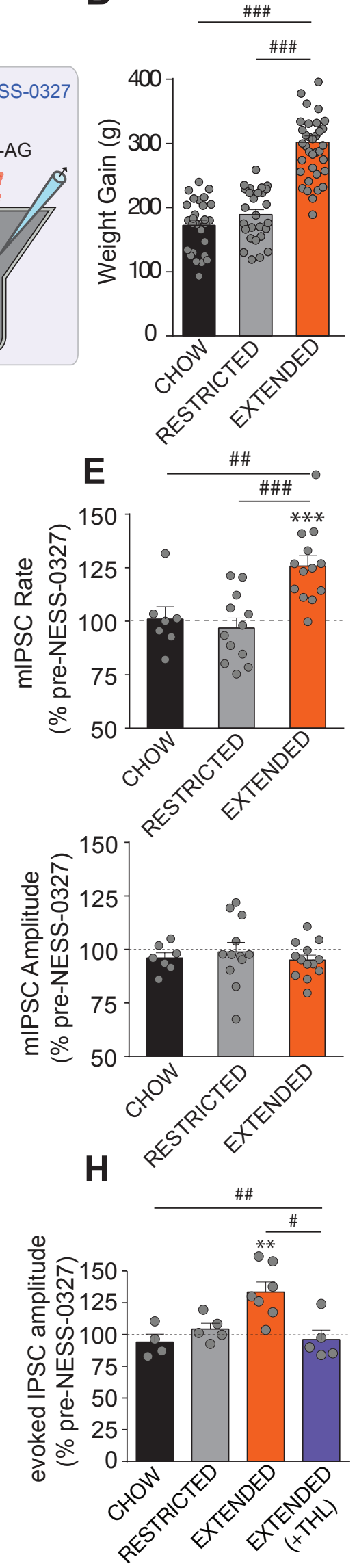

Figure 1 
A

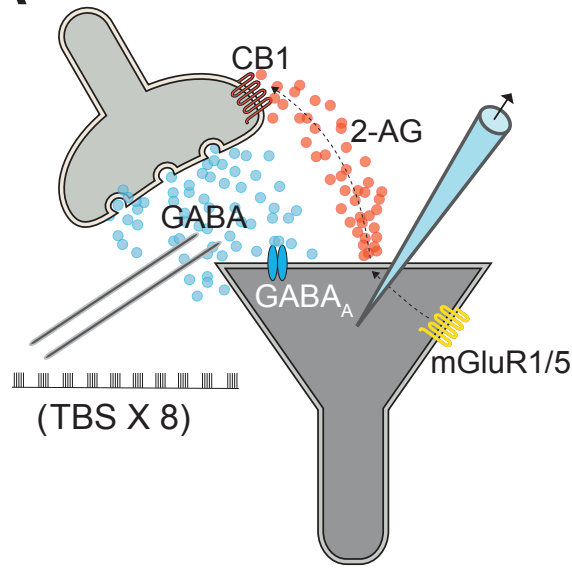

C

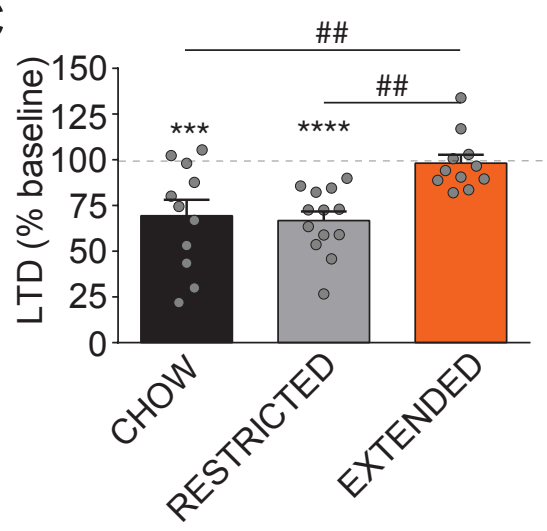

B<smiles>O[C@H]1C=C[C@H]2C=C[C@H]1C2</smiles>

Baseline TBS

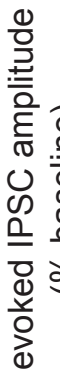

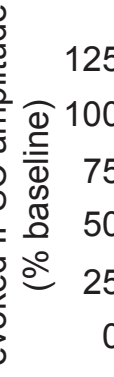

D

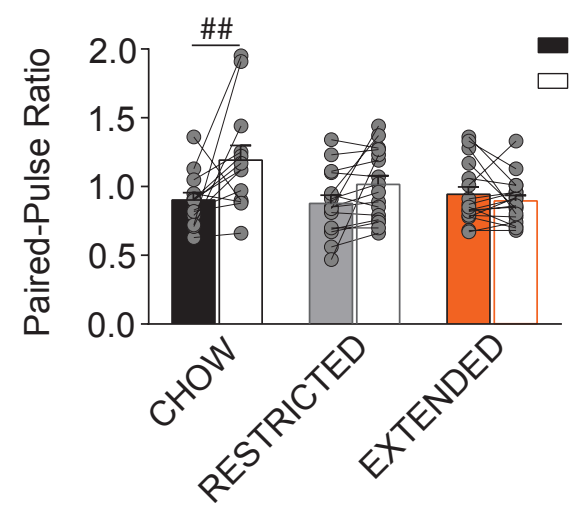

EXTENDED<smiles>CC1CCCC(C)C1C</smiles>

Baseline TBS<smiles>CCCCCCC</smiles>

Baseline TBS 
bioRxiv preprint doi: https://doi.org/10.1101/2020.05.01.073205; this version posted May 3, 2020. The copyright holder for this preprint (which was not certified by peer review) is the author/funder, who has granted bioRxiv a license to display the preprint in perpetuity. It is made available under aCC-BY-NC-ND 4.0 International license.

A

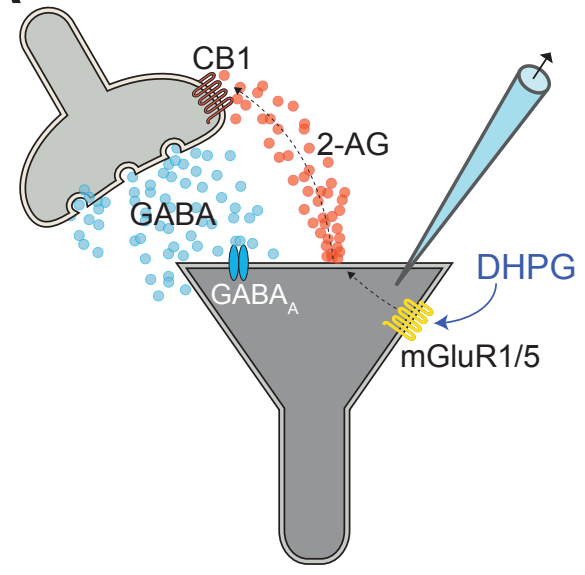

C

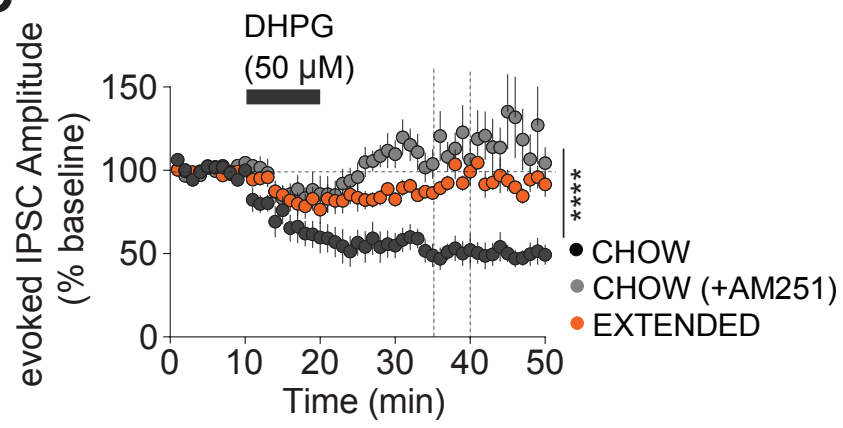

E

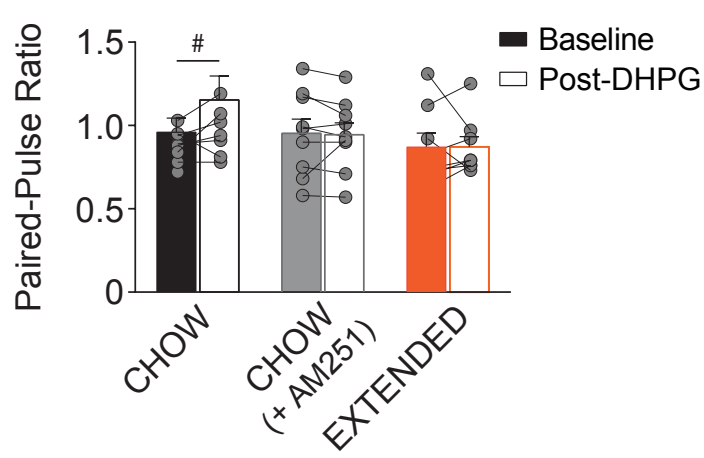

G

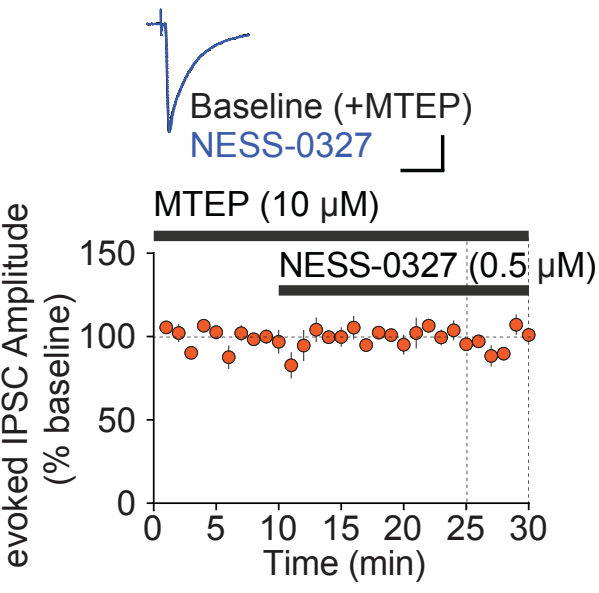

B

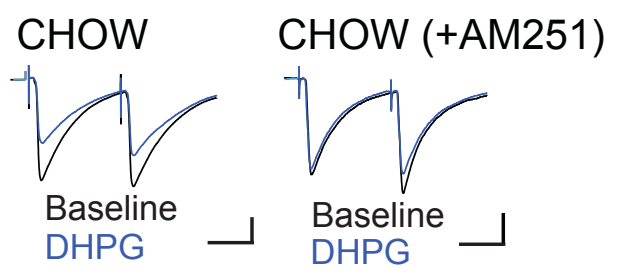

EXTENDED
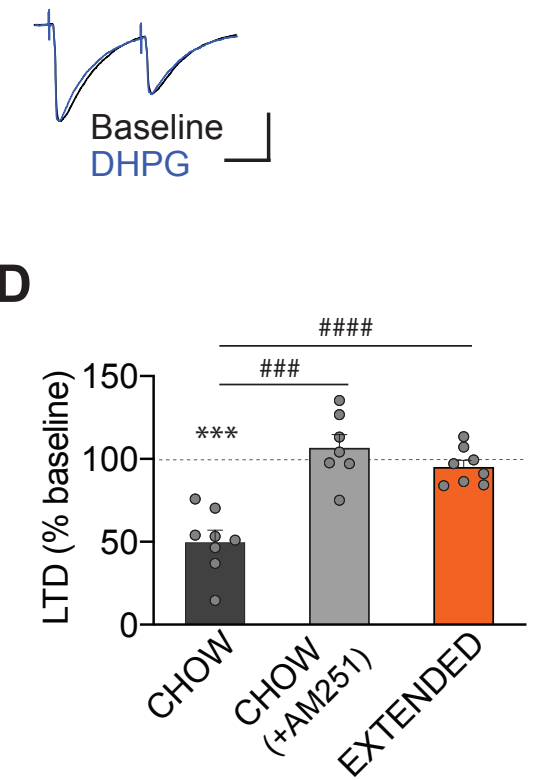

F

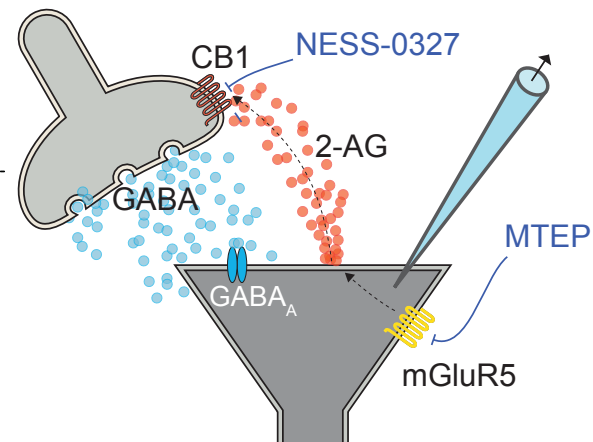

H
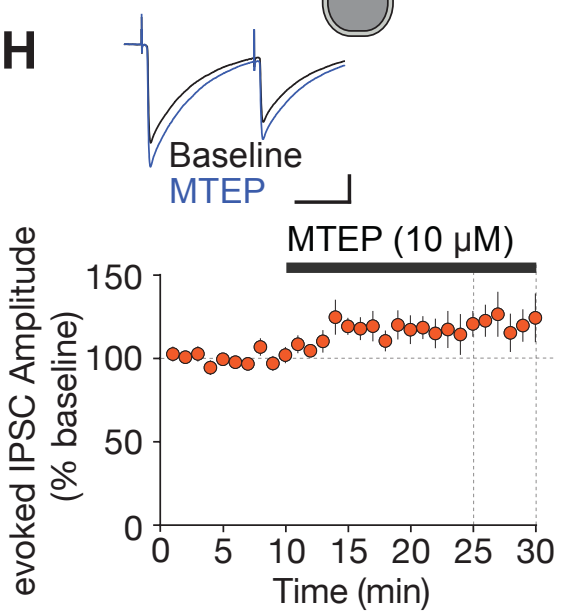
A

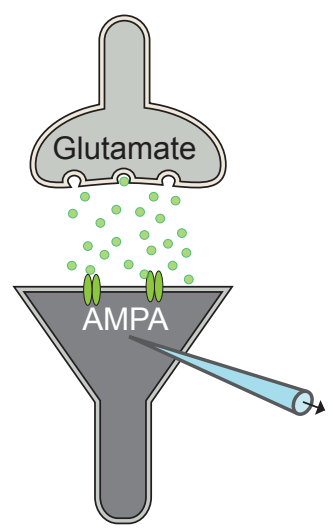

E

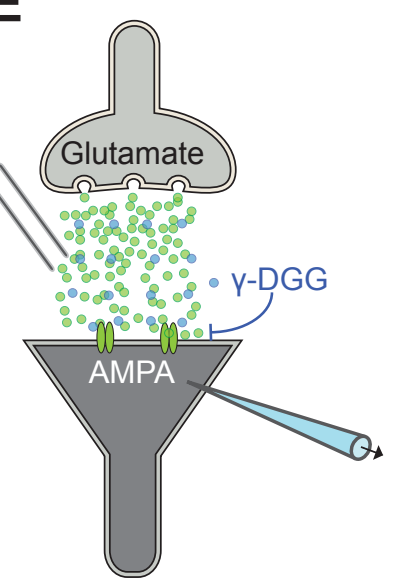

B

CHOW

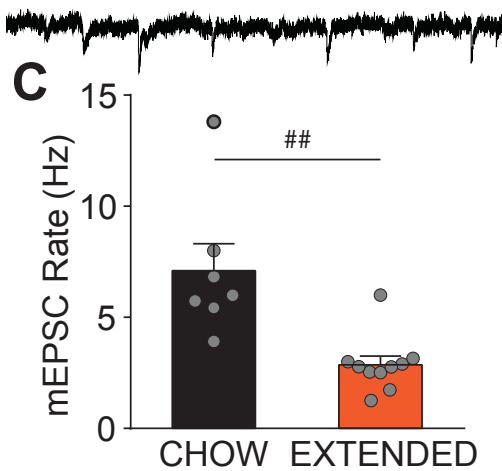

F
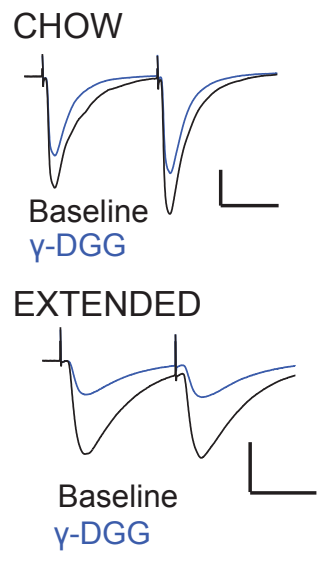

EXTENDED

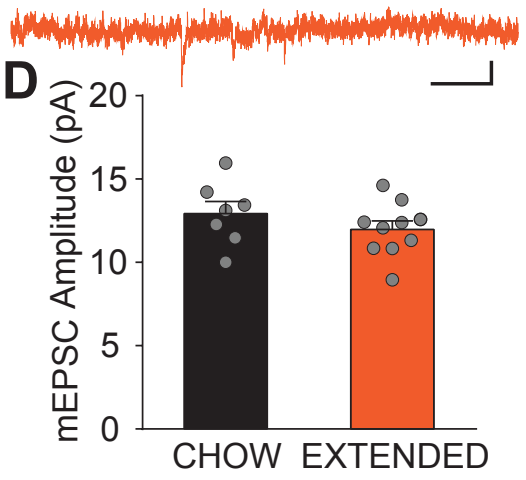

H

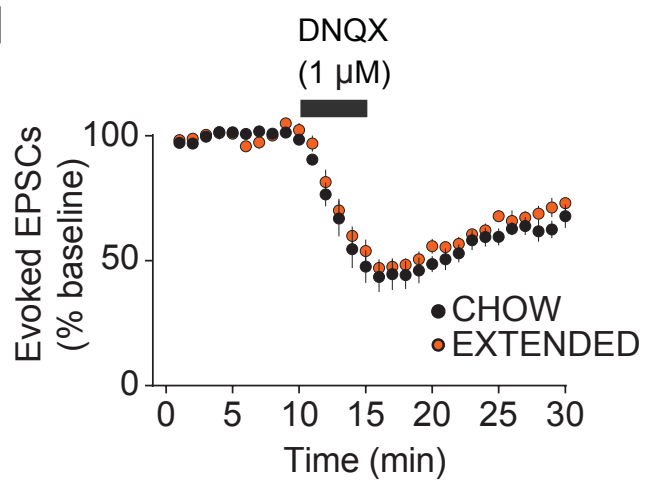

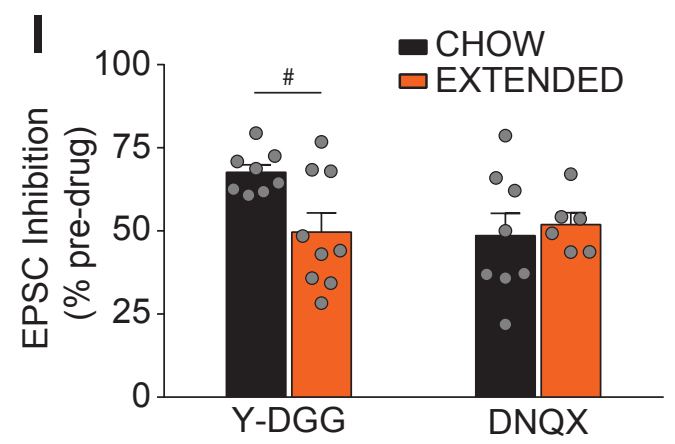

$\mathrm{Y}$-DGG

$(1 \mu \mathrm{M})$

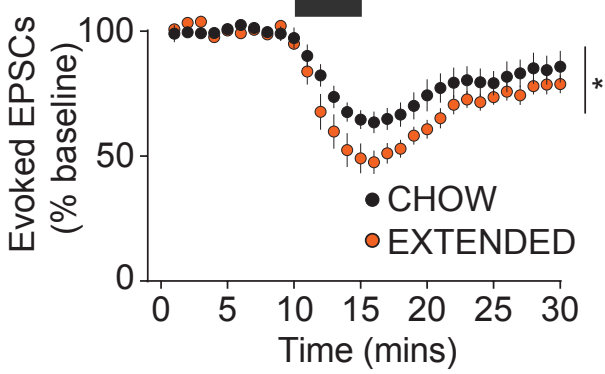

CHOW

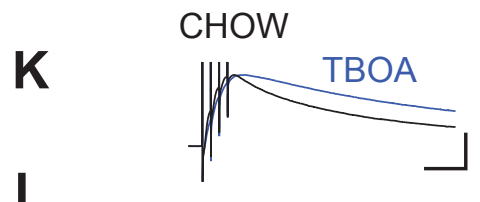

L

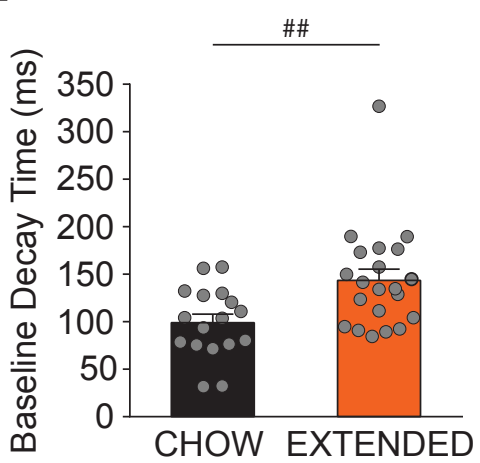

M

EXTENDED
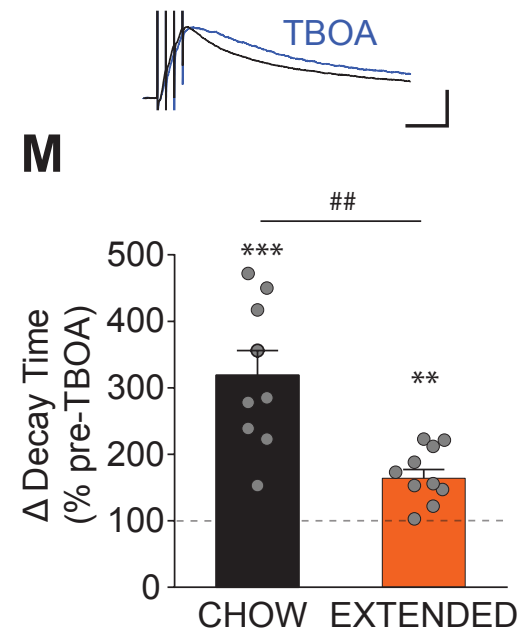
bioRxiv preprint doi: https://doi.org/10.1101/2020.05.01.073205; this version posted May 3, 2020. The copyright holder for this preprint (which was not certified by peer review) is the author/funder, who has granted bioRxiv a license to display the preprint in perpetuity. It is made available under aCC-BY-NC-ND 4.0 International license.

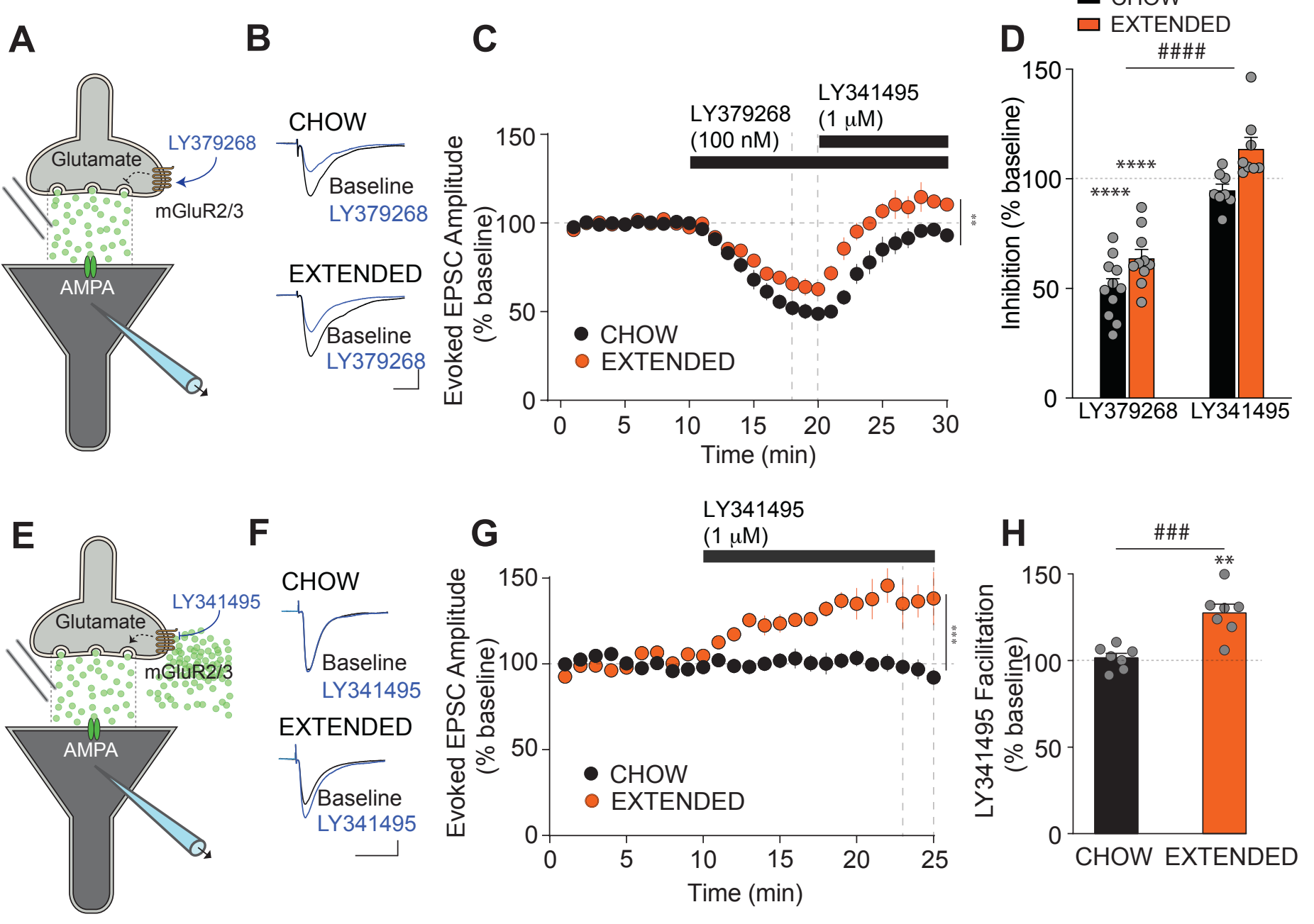

Figure 5 

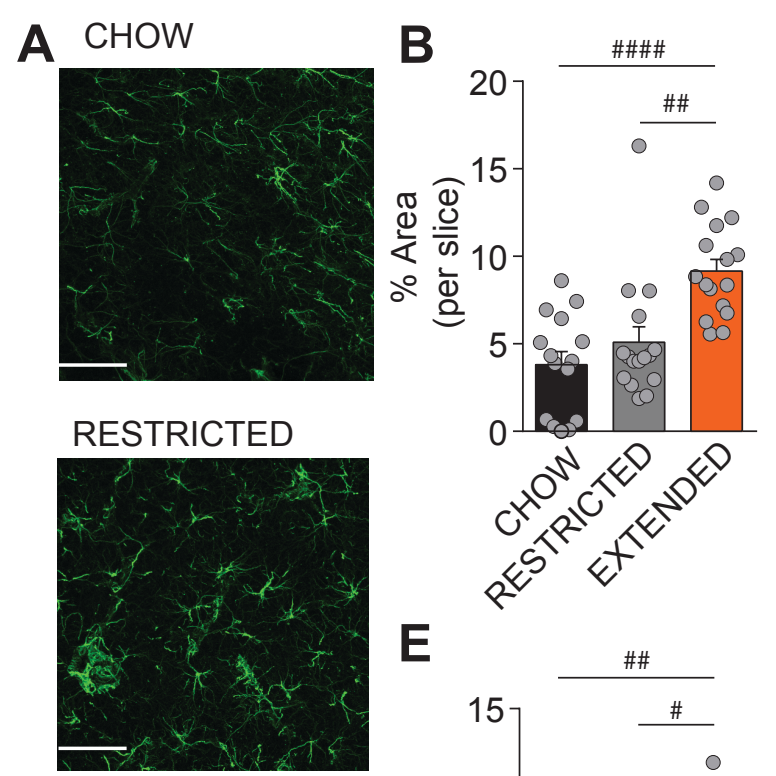

E

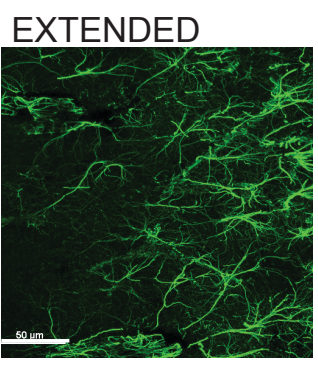

H
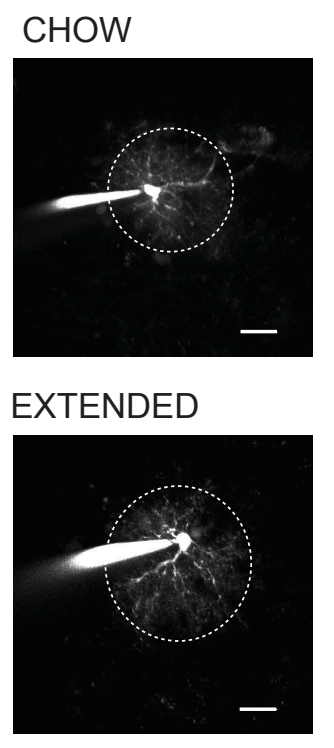

\section{OFC slice}

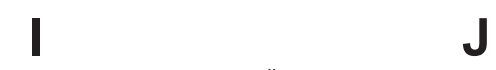

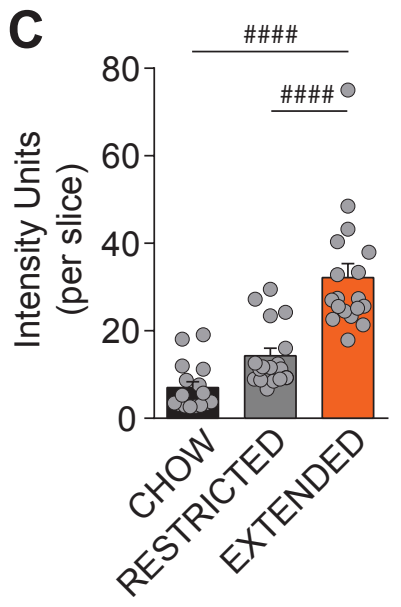

F

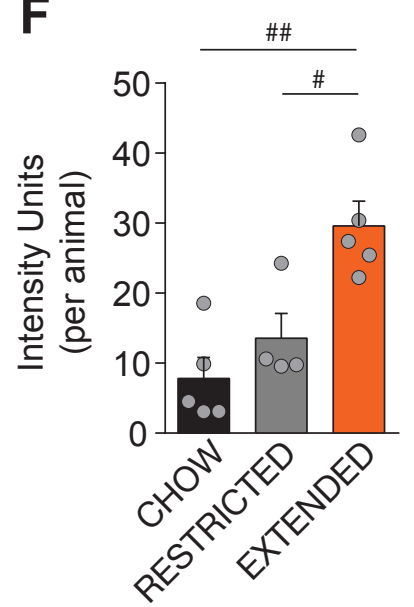

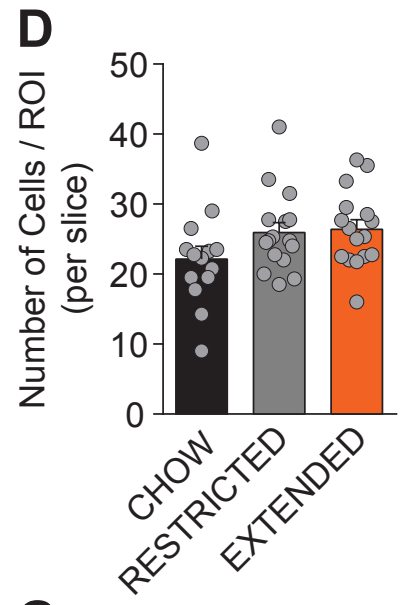

G

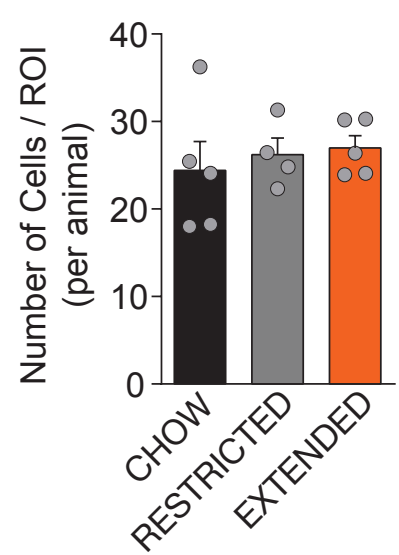

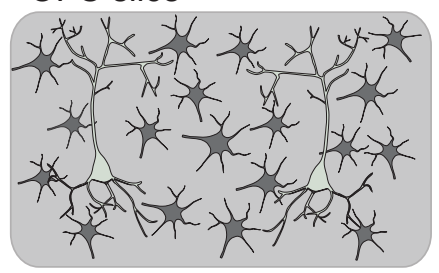

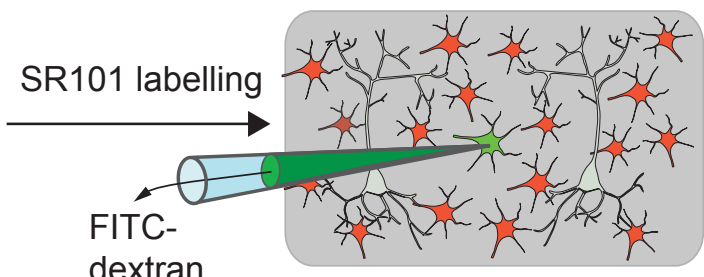

dextran

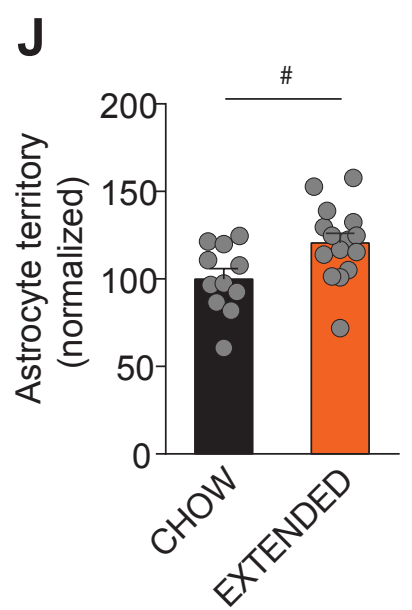

K
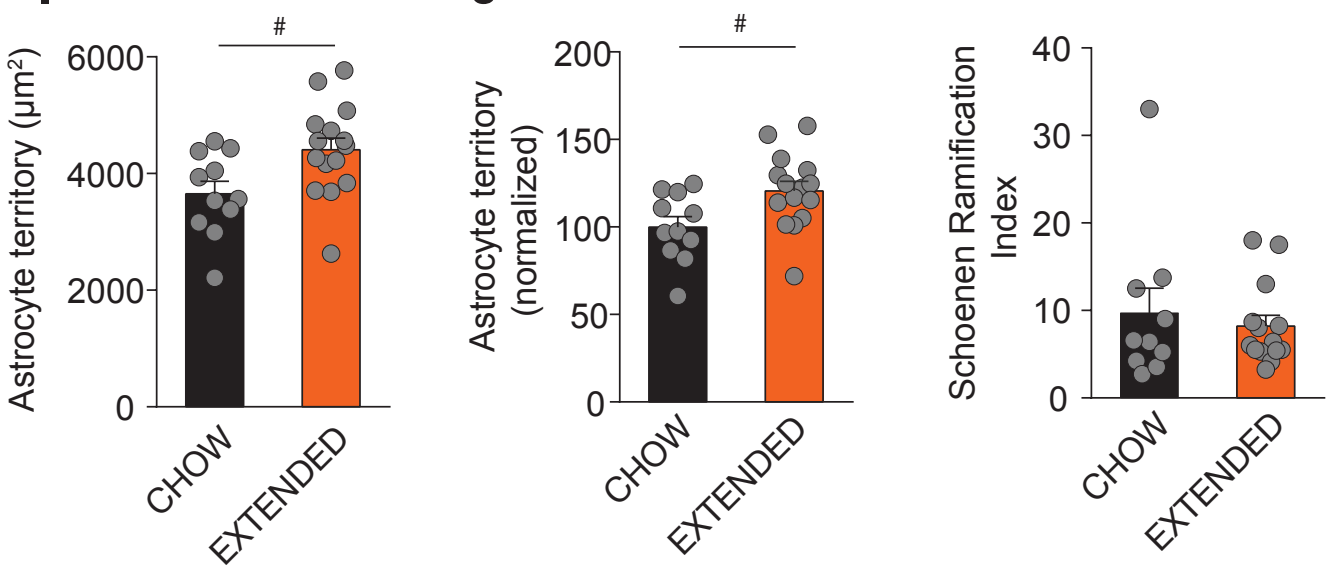

Figure 6 
bioRxiv preprint doi: https://doi.org/10.1101/2020.05.01.073205; this version posted May 3, 2020. The copyright holder for this preprint (which was not certified by peer review) is the author/funder, who has granted bioRxiv a license to display the preprint in perpetuity. It is made available under aCC-BY-NC-ND 4.0 International license.
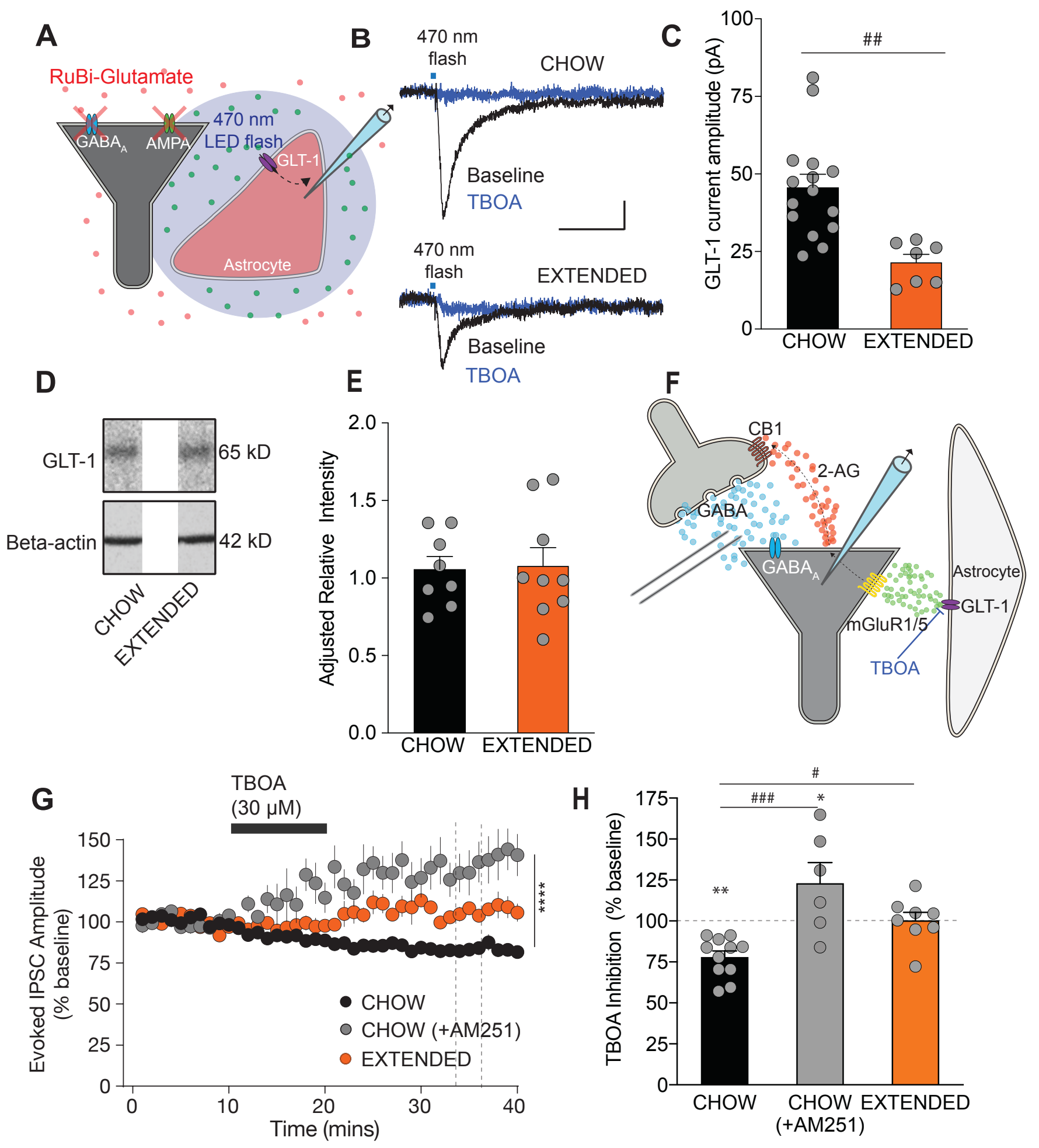
bioRxiv preprint doi: https://doi.org/10.1101/2020.05.01.073205; this version posted May 3, 2020. The copyright holder for this preprint (which was not certified by peer review) is the author/funder, who has granted bioRxiv a license to display the preprint in perpetuity. It is made

A available under aCC-BY-NC-ND 4.0 International license.
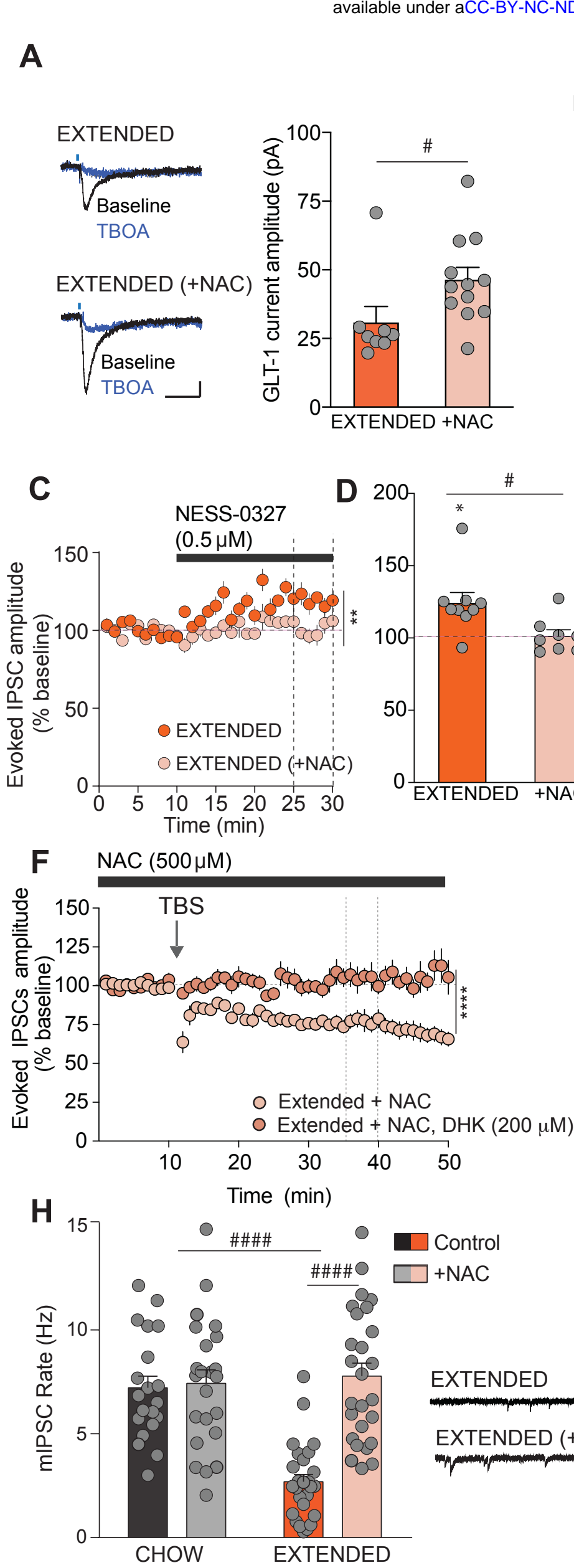

Control

$+N A C$

EXTENDED

EXTENDED (+NAC)

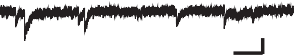

B

EXTENDED

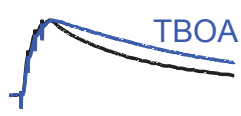

EXTENDED (+ NAC)
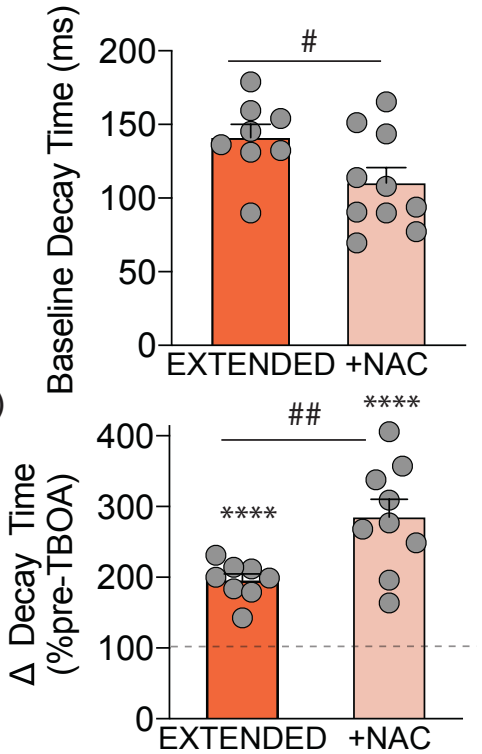

E $\quad \mathrm{NAC}(500 \mu \mathrm{M})$

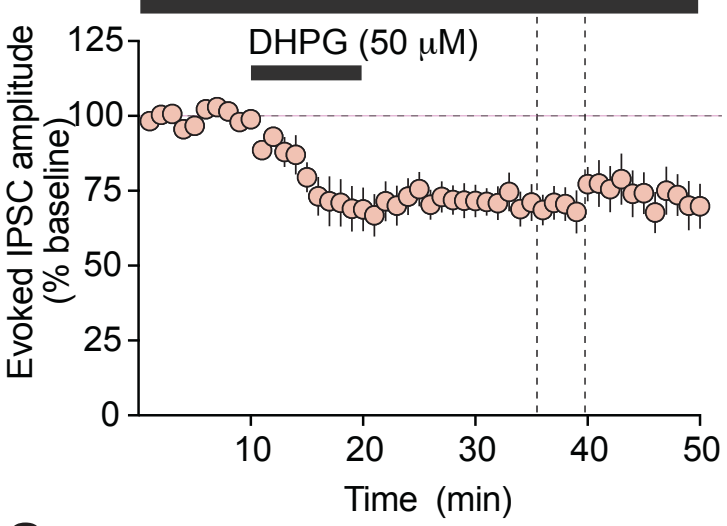

G
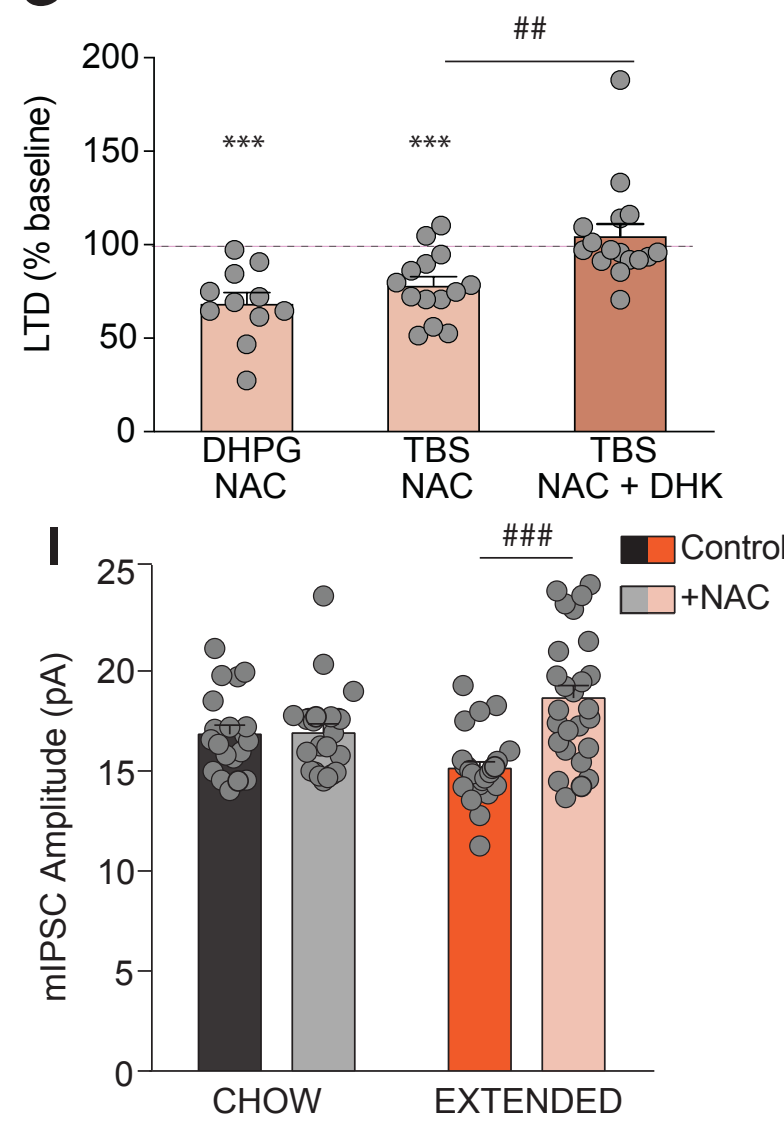

CHOW

Figure 8 
A

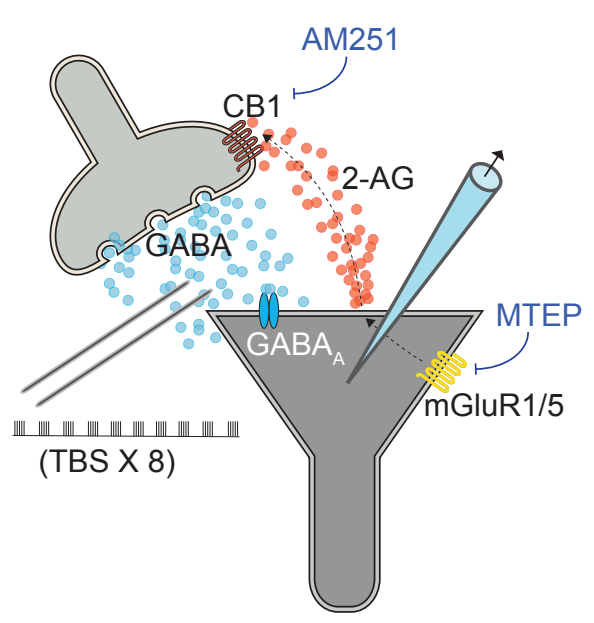

B CONTROL

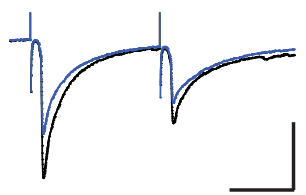

AM251

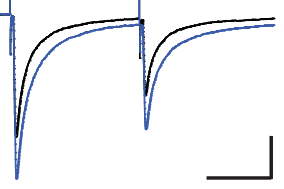

MTEP

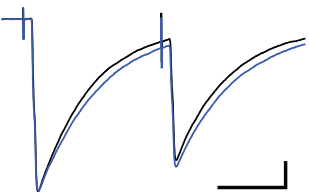

D

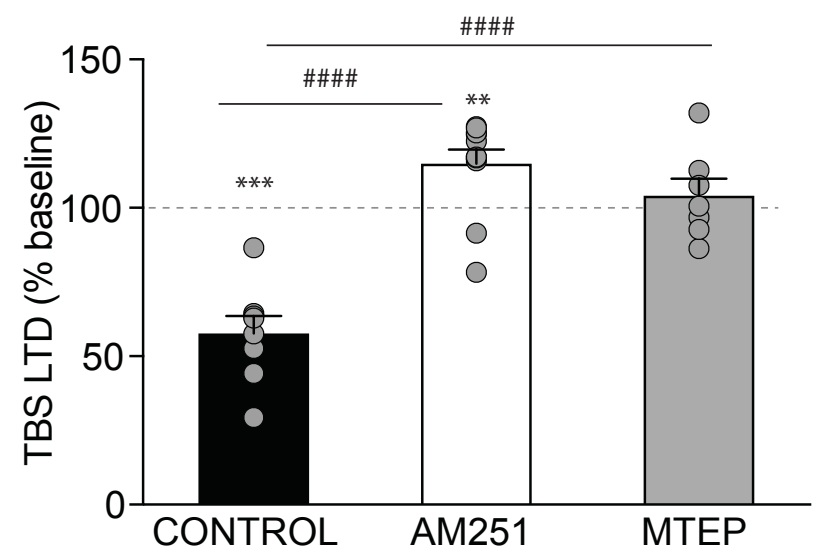

C

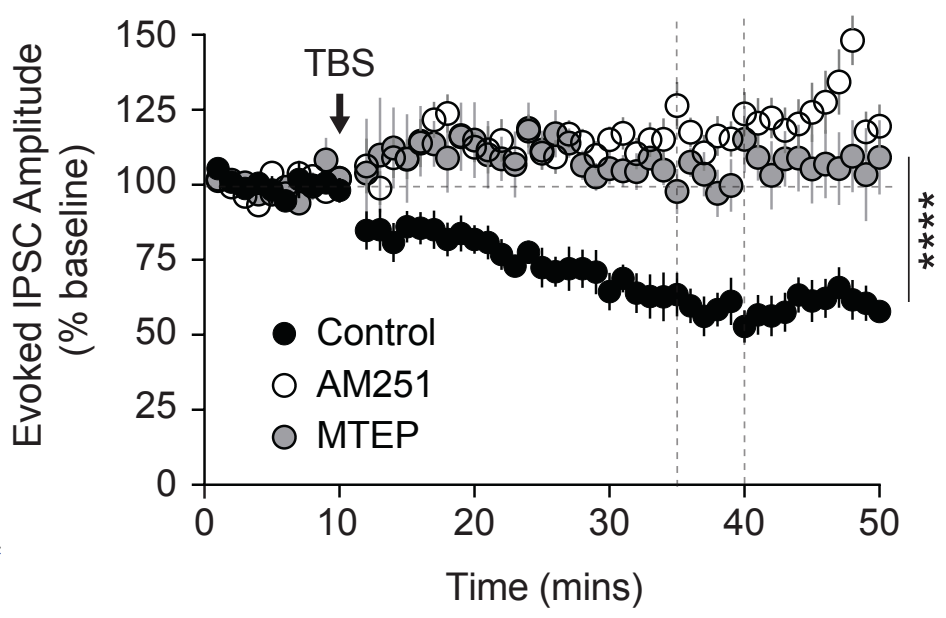

E

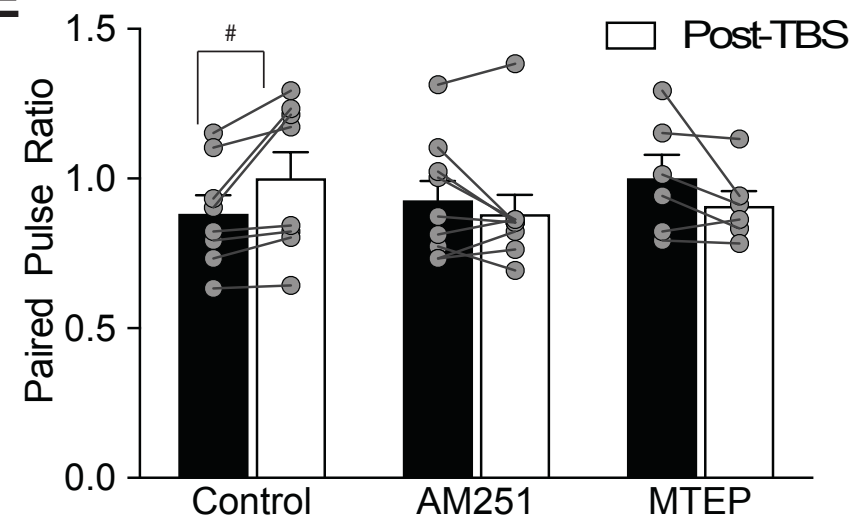

